Pacific

Journal of

Mathematics

WEIGHTED BERGMAN SPACES ON BOUNDED

SYMMETRIC DOMAINS

SALEM Ben SAÏD 


\title{
WEIGHTED BERGMAN SPACES ON BOUNDED SYMMETRIC DOMAINS
}

\author{
SALEM BEN SAÏD \\ To Professor Jacques Faraut on his sixtieth birthday
}

Let $\mathcal{D}$ be a bounded symmetric domain of tube type and $G$ its group of holomorphic automorphisms. In this paper, we describe explicitly the Plancherel Theorem of weighted Bergman spaces on $\mathcal{D}$ under the action of certain symmetric subgroups of $\underline{G}$.

\section{Introduction.}

Let $\underline{G}$ be a noncompact connected real semi-simple Lie group with finite center and Lie algebra $\underline{\mathfrak{g}}$. Let $\theta$ be a Cartan involution of $\underline{G}$ and $\underline{K}=$ $\{g \in \underline{G} \mid \theta(g)=g\}$. We use the same letter $\theta$ to denote the differential of $\theta$. Then, we have a direct sum decomposition $\mathfrak{g}=\underline{\mathfrak{k}} \oplus \mathfrak{p}$ in eigenspaces with respect to $\theta$. We assume that $\underline{G}$ is hermitian, then there exists an element $Z_{0}$ in the center $\mathfrak{c}(\underline{\mathfrak{k}})$ of $\underline{\mathfrak{k}}$ such that $\mathfrak{c}(\underline{\mathfrak{k}})=\mathbb{R} Z_{0}$.

Let $\sigma$ be an involutive automorphism of $\underline{G}$. We may assume that $\sigma$ commutes with $\theta$ and $\mathfrak{g}=\mathfrak{h} \oplus \mathfrak{q}$ is the decomposition of the Lie algebra $\mathfrak{g}$ with respect to $\sigma$. Since $\sigma^{2}=\mathrm{id}$, there are two exclucive possibilites. Either $\sigma\left(Z_{0}\right)=Z_{0}$ and $\sigma$ acts holomorphically on the symmetric domain $\mathcal{D}:=\underline{G} / \underline{K}$, or $\sigma\left(Z_{0}\right)=-Z_{0}$ and $\sigma$ acts anti-holomorphically on $\mathcal{D}$. In this paper we consider the case where $\sigma$ is holomorphic. The case where $\sigma$ is anti-holomorphic is considered by Yu. A. Neretin (cf. [22], [23]). See also [8] and [30].

Let $\mathcal{H}_{\ell}^{2}(\mathcal{D})$ be the ordinary Bergman space of $\mathcal{D}$ where $\mathcal{D}$ is of tube type. For $\nu>\ell-1$, we consider a weighted Bergman space $\mathcal{H}_{\nu}^{2}(\mathcal{D})$ of holomorphic functions on $\mathcal{D}$. The universal covering $\underline{\widetilde{G}}$ of $\underline{G}$ can be realized as the set of pairs $(g, \varphi)$ with $g \in \underline{G}$ and $\varphi$ a holomorphic function on $\mathcal{D}$ where $e^{\varphi(z)}=$ $\operatorname{det}(D g(z))$. Here $D g(z)$ denote the differential of the map $z \mapsto g \cdot z$. The group $\underline{\widetilde{G}}$ acts in $\mathcal{H}_{\nu}^{2}(\mathcal{D})$ by

$$
\left(U_{\nu}(\widetilde{g}) f\right)(z)=e^{\nu \varphi(z)} f(g \cdot z), \quad \widetilde{g}^{-1}=(g, \varphi) .
$$

The representation $U_{\nu}$ is a unitary and irreducible representation.

Let $p$ be the universal map of $\underline{\widetilde{G}}$ in $\underline{G}$, and let $G$ be a symmetric subgroup of $\underline{G}$. In this work we study the decomposition of the restriction of 
$U_{\nu}$ to the subgroup $\widetilde{G}:=p^{-1}(G)$ of $\widetilde{G}$. By [15] (see also [16], [17]) the restriction $U_{\nu \mid \widetilde{G}}$ is decomposed multiplicity-free and discretely into irreducible representations $\left(\pi_{\mu}, \mathcal{H}_{\mu}\right)$ of $\widetilde{G}$ such that $\mathcal{H}_{\mu} \subset \mathcal{H}_{\nu}^{2}(\mathcal{D})$.

Let $\mathcal{S}$ be the Shilov boundary of $\mathcal{D}$. The action of the group $G$ on $\mathcal{S}$ admits open orbits. We consider one of the orbits which is a causal symmetric space $G / H$ of compact type. Moreover $G / H$ is a symmetric Makarevič space. The geometry and analysis of the domain $\mathcal{D}$ and the Makarevič space $G / H$ can be described using Jordan algebras.

To study the decomposition of $\mathcal{H}_{\nu}^{2}(\mathcal{D})$, we consider a $G$-invariant domain $\Xi$ in the complexification $G_{\mathbb{C}} / H_{\mathbb{C}}$ of $G / H$ introduced by J. Hilgert, B. Ørsted and G. Ólafsson (cf. [14]). A geometric descripition of the domain $\Xi$ is given by W. Bertram. The domain $\Xi$ can be realized as $\mathcal{D} \backslash \Sigma$ where $\Sigma$ is an analytic set (cf. [3]).

We consider a covering $\widetilde{\Xi}$ of $\Xi$ with infinite order. We show that there is a unitary isomorphism of $\mathcal{H}_{\nu}^{2}(\mathcal{D})$ onto a weighted Bergman space $\mathcal{H}_{\nu}^{2}(\widetilde{\Xi})$. It is a Hilbert space of holomorphic functions on $\widetilde{\Xi}$, which satisfy a monodromy condition and are square integrable with respect to a $G$-invariant measure on $\Xi$.

To describe explicitly the decomposition of $\mathcal{H}_{\nu}^{2}(\widetilde{\Xi})$ into irreducible subspaces we study the holomorphic discrete series of the universal covering $\widetilde{G}$. Our approach is based on the spherical Laplace transform associated with the ordered symmetric space $G^{c} / H$ dual of $G / H$. See [1] for $G / H \simeq U(p, q)$ and $[2]$ for $G / H$ of Cayley type.

This paper is organized as follows: In Section 2, we give a geometric descripition of the covering $\widetilde{\Xi}$ of $\Xi$ using the theory of Jordan algebras. In Section 3, we study the Bergman space $\mathcal{H}_{\nu}^{2}(\widetilde{\Xi})$ and its reproducing kernel and we establish a unitary isomorphism of $\mathcal{H}_{\nu}^{2}(\widetilde{\Xi})$ onto $\mathcal{H}_{\nu}^{2}(\mathcal{D})$. To describe explicitly the spectrum of $\mathcal{H}_{\nu}^{2}(\widetilde{\Xi})$ and to express its reproducing kernel as series of spherical functions associated with the ordered symmetric spaces $G^{c} / H$, we study in Section 4 the holomorphic discrete series of $\widetilde{G}$. In particular, we obtain a necessary condition for $\pi_{\mu}$ to appear in the Plancherel formula. In Section 5, we compute explicitly the $L^{2}$-norm of matrix coefficient associated with an $H$-spherical unitary highest weight representation. Then, we can state an explicit Plancherel Theorem. The case $G=\underline{K}$ is due to W. Schmid (cf. [28]). See also [11], [12], [26] and [29].

\section{Geometric realization of the covering $\widetilde{\Xi}$.}

Let $\mathbb{V}$ be a Euclidean Jordan algebra, and let $\Omega$ be the associated symmetric cone. We denote the dimension of $\mathbb{V}$ by $n$, the rank by $r$, and the unit element 
by $e$. A Euclidean Jordan algebra is said to be simple if it has no nontrivial ideal (cf. [12], Chapter II).

Let $\mathcal{D}$ be the unit disc of $\mathbb{V}_{\mathbb{C}}:=\mathbb{V}+i \mathbb{V}$ with respect to the spectral norm

$$
\mathcal{D}:=\left\{z \in \mathbb{V}_{\mathbb{C}} \mid e-z \square \bar{z} \gg 0\right\},
$$

where $z_{\square} w:=L(z w)+[L(z), L(w)]$. Here $L(z)$ denotes the endomorphism of $\mathbb{V}_{\mathbb{C}}$ defined by $L(z) w=z w$.

Let $\underline{G}$ be the group of holomorphic automorphisms of $\mathcal{D}$ and let $\underline{K}$ be the isotropy subgroup of 0 in $\underline{G}$. It is a maximal compact subgroup of $\underline{G}$. The Lie algebra $\mathfrak{g}$ of $\underline{G}$ is consists of vector fields of the form

$$
X(z)=w+T z-P(z) \bar{w},
$$

where $w \in \mathbb{V}_{\mathbb{C}}, T \in \underline{\mathfrak{k}}:=\operatorname{Lie}(\underline{K})$ and $P(z):=2 L(z)^{2}-L\left(z^{2}\right)$. The application $P$ is called a quadratic representation associated with $\mathbb{V}_{\mathbb{C}}$. We identify a vector field $X$ with the triplet $(w, T, \bar{w})$.

Let $\alpha$ be an involutive automorphism of the Jordan algebra $\mathbb{V}$. Denote also $\alpha$ its $\mathbb{C}$-linear extention to $\mathbb{V}_{\mathbb{C}}$. The Jordan algebra $\mathbb{V}$ and its complexification $\mathbb{V}_{\mathbb{C}}$ decompose into eigenspaces with respect to the involution $\alpha$

$$
\mathbb{V}=\mathbb{V}^{+} \oplus \mathbb{V}^{-}, \quad \mathbb{V}_{\mathbb{C}}=\mathbb{V}_{\mathbb{C}}^{+} \oplus \mathbb{V}_{\mathbb{C}}^{-}
$$

We say that the pair $(\mathbb{V}, \alpha)$ is irreducible if it is not possible to write

$$
(\mathbb{V}, \alpha)=\left(\mathbb{V}_{1} \oplus \mathbb{V}_{2}, \alpha_{1} \oplus \alpha_{2}\right) .
$$

We show that if $(\mathbb{V}, \alpha)$ is irreducible then either $\mathbb{V}$ is simple, or $\mathbb{V}=\mathbb{V} \circ \times \mathbb{V}$ 。 where $\mathbb{V}_{\circ}$ is a simple Euclidean Jordan algebra and $\alpha(x, y)=(-y,-x)$. We note that $\mathbb{V}^{+}$is either simple or a direct sum of two simple algebras.

Let $\left\{c_{1}, \ldots, c_{r}\right\}$ be a Jordan frame of $\mathbb{V}$. It is a complete system of orthogonal primitive idempotent elements. The algebra $R:=\bigoplus_{j=1}^{r} \mathbb{R} c_{j}$ is a maximal associative subalgebra of $\mathbb{V}$. Assume $\alpha$ is given such that $\alpha(R)=R$, then $R=R^{+} \oplus R^{-}$is the decomposition of $R$ into eigenspaces with respect to $\alpha$. We note $r^{+}:=\operatorname{dim} R^{+}$.

Theorem 2.1 (cf. [4]). Let $\mathbb{V}$ be a Euclidean Jordan algebra and let $\alpha$ be an involutive automorphism of $\mathbb{V}$.

(1) The rank of the Euclidean Jordan algebra $\mathbb{V}^{+}$is equal to $r^{+}$.

(2) Either $R=R^{+}$and $r=r^{+}$, or $r=2 r^{+}$and $\operatorname{dim} R^{+}=\operatorname{dim} R^{-}$.

Let

$$
\underline{G}^{(-\alpha)}:=\{g \in \underline{G} \mid(-\alpha) \circ g \circ(-\alpha)=g\},
$$

and let $G$ be its connected identity component. In particular if $\alpha=\mathrm{id}_{\mathbb{V}}$ then $G=\underline{K}$. 
The Lie algebra $\mathfrak{g}$ of $G$ is consists of vector fields $X$ on $\mathbb{V}_{\mathbb{C}}$ such that $(-\alpha) \circ$ $X \circ(-\alpha)=X$. Then $\mathfrak{g}$ is isomorphic to the set of triplets

$$
\left\{(w, T, \bar{w}) \mid w \in \mathbb{V}_{\mathbb{C}}^{-}, T \in \underline{\mathfrak{k}} \text { and } \alpha \circ T \circ \alpha=T\right\} .
$$

We write, for $z \in \mathbb{V}_{\mathbb{C}}, j(z):=z^{-1}$ the inverse of $z$ in the Jordan algebra $\mathbb{V}_{\mathbb{C}}$, and $\tau$ the conjugation of $\mathbb{V}_{\mathbb{C}}$ with respect to the real form $\mathbb{V}$. The application $\theta: g \mapsto(-j \tau) \circ g \circ(-j \tau)$ is a Cartan involution of the Lie algebra $\mathfrak{g}$ (cf. [3]). Then

$$
\begin{aligned}
& \mathfrak{k}:=\mathfrak{g}^{\theta}=\{(0, T, 0) \mid T \in \underline{\mathfrak{k}} \text { and } \theta \circ T \circ \theta=T\}, \\
& \mathfrak{p}:=\mathfrak{g}^{-\theta}=\left\{(w, 0, \bar{w}) \mid w \in \mathbb{V}_{\mathbb{C}}^{-}\right\} .
\end{aligned}
$$

Let $H$ be the stabilizer of the base point $i e$ in $G$,

$$
H:=\{g \in G \mid g \cdot(i e)=i e\} .
$$

Proposition 2.1. The pair $(G, H)$ is a symmetric pair.

Proof. Let $\sigma$ be the involution of $G$ defined by

$$
\sigma(g)=(-j) \circ g \circ(-j),
$$

which commutes with the Cartan involution $\theta$ defined before. The differential of $\sigma$, also denoted by $\sigma$, is given by

$$
\sigma(w, T, \bar{w})=\left(-\bar{w},-T^{\prime},-w\right)
$$

where $T^{\prime}$ denote the adjoint of $T$ with respect to the scalar product on $\mathbb{V}$ defined by the trace. By definition of $H$, its Lie algebra $\mathfrak{h}$ is given by

$$
\mathfrak{h}=\left\{(i w, T, i w) \mid w \in \mathbb{V}^{-}, T \in \operatorname{Der}\left(\mathbb{V}^{+}\right)\right\},
$$

where $\operatorname{Der}\left(\mathbb{V}^{+}\right)$is the derivation algebra of $\mathbb{V}^{+}$. Then

$$
\mathfrak{h}=\mathfrak{g}^{\sigma}:=\{X \in \mathfrak{g} \mid \sigma(X)=X\} .
$$

The pseudo-Riemannian symmetric space $G / H$ is the open orbit $G \cdot i e$ in the Shilov boundary of $\mathcal{D}$. It is a compactly causal symmetric space. Moreover $G / H$ is a Makarevič symmetric space (cf. [3], [21]). With respect to the involution $\sigma$, the Lie algebra $\mathfrak{g}$ decomposes as $\mathfrak{g}=\mathfrak{h} \oplus \mathfrak{q}$ where $\mathfrak{q}:=$ $\mathfrak{g}^{-\sigma}=\left\{(w, i L(v), w) \mid w \in \mathbb{V}^{-}, v \in \mathbb{V}^{+}\right\}$.

The Lie algebra $\mathfrak{g}$ is semisimple and hermitien. By a theorem of Vinberg and Kostant, there is a regular $\underline{G}$-invariant cone (i.e., convex, closed, proper, and with nonempty interior) in $\mathfrak{g}$. Let $C_{\max }$ be a maximal regular $\underline{G}$-invariant cone in $\underline{\mathfrak{g}}$ containing $(0, i I, 0)$. By $[\mathbf{2 5}]$,

$$
\Gamma\left(C_{\text {max }}\right):=\underline{G} \exp \left(i C_{\text {max }}\right)=\left\{g \in \underline{G}_{\mathbb{C}} \mid g \cdot \overline{\mathcal{D}} \subset \mathcal{D}\right\} .
$$

In the complexified space $G_{\mathbb{C}} / H_{\mathbb{C}}$ of $G / H$ we consider the complex domain

$$
\Xi:=\Gamma\left(C^{0}\right) \cdot i e
$$


where $C^{0}$ is the interior of $C:=i\left(C_{\max } \cap \mathfrak{g}\right)$. (This domain is introduced by J. Hilgert, B. Ørsted, and G. Ólafsson in [14].)

The domain $\Xi$ can be realized as $\mathcal{D} \backslash \Sigma$ where $\Sigma$ is the analytic set given by

$$
\Sigma=\{z \in \mathcal{D} \mid \operatorname{det}(P(z+\alpha z))=0\},
$$

where the notation "det" denotes the determinant with respect to $\mathbb{V}$ (cf. [3]).

The domain $\Xi$ can also be realized as a subset of the imaginary tangent bundle of $G / H$

$$
\Xi \simeq G \times C^{\mathfrak{q}} / \sim
$$

where $C^{\mathfrak{q}}:=C^{0} \cap i \mathfrak{q}$, and $G \times C^{\mathfrak{q}} / \sim$ is the quotient of $G \times C^{\mathfrak{q}}$ by the equivalence relation: $\left(g_{1}, X_{1}\right) \sim\left(g_{2}, X_{2}\right)$ if and only if there exists $h \in H$ such that

$$
g_{2}=g_{1} h \quad \text { and } \quad X_{2}=\operatorname{Ad}\left(h^{-1}\right) X_{1}
$$

(cf. $[14])$.

The open set $\Xi$ is connected since it is homeomorphic to $\mathcal{D} \backslash \Sigma$, observing that $\mathcal{D}$ is connected and $\operatorname{codim}_{\mathbb{R}}(\Sigma)=2$.

Let

$$
\widetilde{\Xi}:=\left\{(z, \zeta) \in \Xi \times \mathbb{C} \mid e^{\frac{2 n}{r^{+}} \zeta}=\operatorname{det}(P(z+\alpha z))\right\} .
$$

Note that $\frac{2 n}{r^{+}}$is an integer.

Theorem 2.2. The set $\widetilde{\Xi}$ is a connected covering of infinite order of the domain $\Xi$.

Proof. Let $p$ be the map defined by

$$
\begin{aligned}
& p: \widetilde{\Xi} \longrightarrow \Xi, \\
& (z, \zeta) \mapsto z .
\end{aligned}
$$

Then $p$ is surjective. In fact for $z \in \Xi$, we have $\operatorname{det}(P(z+\alpha z)) \neq 0$, then there exists $\zeta \in \mathbb{C}$ such that $e^{\frac{2 n}{r^{+}} \zeta}=\operatorname{det}(P(z+\alpha z))$. Let $z_{0} \in \Xi$, we can find an open neighbourhood $U$ of $z_{0}$ such that $p^{-1}(U)$ is homeomorphic to $U \times \mathbb{Z}$. In fact, since $p$ is surjective, there exists $\left(z_{0}, \zeta_{0}\right) \in \widetilde{\Xi}$ such that $p\left(z_{0}, \zeta_{0}\right)=z_{0}$. We consider a determination of $\log (\operatorname{det}(P(z+\alpha z)))$ in the neighbourhood $U$ of $z_{0}$, we can define a homeomorphism of $U \times \mathbb{Z}$ in $p^{-1}(U)$ as

$$
(z, m) \mapsto(z, \log (\operatorname{det}(P(z+\alpha z)))+2 \pi i m) .
$$

Hence $\widetilde{\Xi}$ is a covering of infinite order of $\Xi$. 
Let $\left\{e_{1}, e_{2}, \ldots, e_{r^{+}}\right\}$be a Jordan frame of $\mathbb{V}^{+}$. An element $z$ of the form $z=\sum_{j=1}^{r^{+}} z_{j} e_{j}$ belongs to $\Xi$ if and only if $0<\left|z_{j}\right|<1$. Let

$$
z(t)=\sum_{j=1}^{r^{+}-1} e_{j}+e^{2 \pi i t} e_{r^{+}} \in \bar{\Xi}
$$

and

$$
z_{0}=\sum_{j=1}^{r^{+}} z_{j} e_{j} \in \Xi
$$

The curve $\varphi(t):=z(t) z_{0}$ belongs to $\Xi$ and satisfies $\varphi(0)=\varphi(1)=z_{0}$. Let $\widetilde{\varphi}$ be the lifting of $\varphi$ to $\widetilde{\Xi}$,

$$
\begin{aligned}
\widetilde{\varphi}:[0,1] & \longrightarrow \widetilde{\Xi}, \\
t & \mapsto(\varphi(t), \zeta(t)) .
\end{aligned}
$$

Using the fact that $e_{j}=c_{j}$ if $r=r^{+}$and $e_{j}=c_{j}+c_{j+r^{+}}$if $r=2 r^{+}$, for all $1 \leq j \leq r^{+}$, we deduce that

$$
\begin{aligned}
e^{\frac{2 n}{r^{+}} \zeta(t)} & =\operatorname{det}(P(z(t)+\alpha z(t))) \\
& =C\left(z_{1}, \ldots, z_{r^{+}}\right)\left(e^{2 \pi i t}\right)^{\frac{2 n}{r^{+}}},
\end{aligned}
$$

where $C\left(z_{1}, \ldots, z_{r^{+}}\right)$is a nonzero constant depending on $z_{1}, \ldots, z_{r^{+}}$. There exists $\hbar \in \mathbb{C}^{*}$ such that $C\left(z_{1}, \ldots, z_{r^{+}}\right)=e^{\hbar}$ and $\frac{2 n}{r^{+}}(\zeta(t)-2 \pi i t)=\hbar+$ $2 \pi i \kappa(t)$. Here $\kappa(t)$ is an integer valued continuous function on $[0,1]$, therefore constant. Thus $\zeta(1)-\zeta(0)=2 \pi i$ and if $\widetilde{\varphi}(0)=\left(z_{0}, \zeta_{0}\right)$, then $\widetilde{\varphi}(1)=$ $\left(z_{0}, \zeta_{0}+2 \pi i\right)$. Thus if $z_{0}$ is an element of $\Xi \cap \bigoplus_{j=1}^{r^{+}} \mathbb{C} e_{j}$ and if $\left(z_{0}, \zeta_{0}^{1}\right)$ and $\left(z_{0}, \zeta_{0}^{2}\right)$ are two points of $\widetilde{\Xi}$, there exists a curve $\widetilde{\varphi}$ such that $\widetilde{\varphi}(0)=\left(w_{0}, \zeta_{0}^{1}\right)$ and $\widetilde{\varphi}(1)=\left(z_{0}, \zeta_{0}^{2}\right)$.

Let $\left(z_{1}, \zeta_{1}\right)$ and $\left(z_{2}, \zeta_{2}\right)$ be two points of $\widetilde{\Xi}$. Since $\Xi$ is connected, there exists a curve $\varphi_{1}$ (resp. $\left.\varphi_{2}\right)$ such that

$$
\varphi_{1}(0)=z_{0}, \quad \varphi_{1}(1)=z_{1} \quad\left(\operatorname{resp} . \quad \varphi_{2}(0)=z_{0}, \quad \varphi_{2}(1)=z_{2}\right) .
$$

Let $\widetilde{\varphi_{1}}$ (resp. $\left.\widetilde{\varphi_{2}}\right)$ be the lifting of $\varphi_{1}\left(\right.$ resp. $\left.\varphi_{2}\right)$ to $\widetilde{\Xi}$ such that

$$
\begin{array}{ll}
\widetilde{\varphi_{1}}(0)=\left(z_{0}, \zeta_{0}^{1}\right) & \widetilde{\varphi_{1}}(1)=\left(z_{1}, \zeta_{1}\right) \\
\widetilde{\varphi_{2}}(0)=\left(z_{0}, \zeta_{0}^{2}\right) & \widetilde{\varphi_{2}}(1)=\left(z_{2}, \zeta_{2}\right) .
\end{array}
$$

Using the fact that $z_{0} \in \Xi \cap \bigoplus_{j=1}^{r^{+}} \mathbb{C} e_{j}$, we deduce that $\widetilde{\Xi}$ is connected. 
Let

$$
\top_{\alpha}(z)=\operatorname{det}(P(z+\alpha z))
$$

With respect to Lebesgue measure, the restriction to $\Xi$ of the $G_{\mathbb{C} \text {-invariant }}$ measure of $G_{\mathbb{C}} / H_{\mathbb{C}}$ is given by

$$
d \xi=\frac{d \lambda(z)}{\left|T_{\alpha}(z)\right|}
$$

Let

$$
\top_{\alpha}(\widetilde{z})^{\frac{r}{4 n} \nu}:=e^{\frac{r}{2 r^{+}} \nu \zeta}, \quad \widetilde{z}=(z, \zeta) \in \widetilde{\Xi}
$$

The realization of the domain $\Xi$ inside the imaginary tangent bundle of $G / H$ will be used below which permits to show an integral formula.

Let $\mathfrak{a}$ be a Cartan subalgebra in $\mathfrak{k} \cap \mathfrak{q}$. Let $\triangle$ be the root system $\triangle\left(\mathfrak{g}_{\mathbb{C}}, i \mathfrak{a}\right)$, and $\mathfrak{a}^{+}$a positive Weyl chamber. Let $\triangle^{+}$be the positive root system with respect to $i \mathfrak{a}^{+}$.

Theorem 2.3. For an integrable function $f$ on $\Xi$,

$$
\int_{\Xi} f(\xi) d \xi=c_{0} \int_{G} \int_{C^{+}} f(g \exp (X) \cdot i e) \prod_{\beta \in \triangle^{+}}(\operatorname{sh}\langle\beta, 2 X\rangle)^{m_{\beta}} d g d X
$$

where $C^{+}:=C^{0} \cap i \mathfrak{a}^{+}$and $m_{\beta}=\operatorname{dim}\left(\mathfrak{g}_{\beta}\right)$.

Proof. Let $Z:=Z_{H}(i \mathfrak{a})$ be the centralizer subgroup of $i \mathfrak{a}$ in $H$. The map

$$
\begin{aligned}
\varphi: G / Z \times C^{+} & \longrightarrow \Xi \\
(g \cdot Z, X) & \mapsto g \exp (X) \cdot i e
\end{aligned}
$$

is a diffeomorphism onto its open image. In fact, let $g_{1} \exp \left(X_{1}\right) \cdot i e$ and $g_{2} \exp \left(X_{2}\right) \cdot i e$ be two elements of $\Xi$ such that $g_{1} \exp \left(X_{1}\right) \cdot i e=g_{2} \exp \left(X_{2}\right) \cdot i e$. Since the group $G$ acts on $G / Z \times C^{+}$(resp. $\Xi$ ) by

$$
g_{0} \cdot(g \cdot Z, X)=\left(g_{0} g \cdot Z, X\right), \quad\left(\text { resp. } g_{0} \cdot(g \exp (X) \cdot i e)=g_{0} g \exp (X) \cdot i e\right),
$$

we may assume that $g_{1}=1$. But since $X_{1}$ and $X_{2}$ are regular and in the same positive Weyl chamber, by the equivalence relation (2), we deduce that $X_{1}=X_{2}$. Thus establishing injectivity. 
We will compute the differential of $\varphi$. For this we consider the commutative diagram defined by

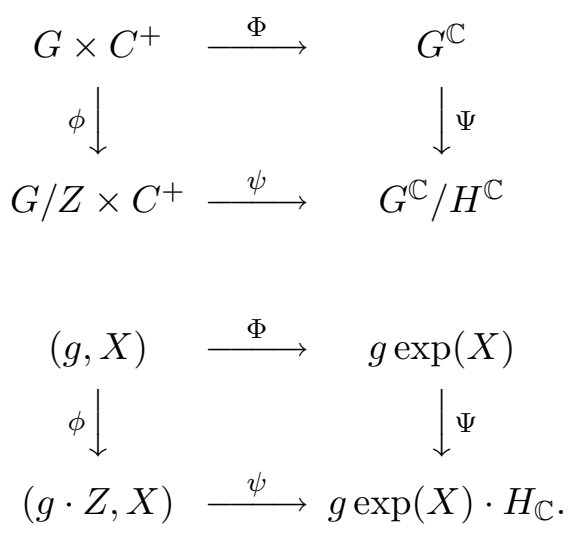

Then $\mathrm{d}(\psi \circ \phi)(1, X)=\mathrm{d}(\Psi \circ \Phi)(1, X)$.

Let $\lambda_{g}\left(\right.$ resp. $\left.\Lambda_{g}\right)$ be the left translation by $g$ in $G$ (resp. $G_{\mathbb{C}} / H_{\mathbb{C}}$ ), then for $(Y, U) \in \mathfrak{g} \times i \mathfrak{a}$,

$$
\begin{aligned}
& \mathrm{d}(\Psi \circ \Phi)(1, X)(Y, U) \\
& =\frac{\mathrm{d}}{\mathrm{d} t}{ }_{t=0} \Psi(\exp (t Y) \exp (X+t U)) \\
& =\mathrm{d} \Psi(\exp (X)) \cdot \mathrm{d} \lambda_{\exp (X)}(1) \cdot\left(e^{-\operatorname{ad}(X)} \cdot Y+\frac{1-e^{-\operatorname{ad}(X)}}{\operatorname{ad}(X)} \cdot U\right) \\
& =\mathrm{d} \Lambda_{\exp (X)} \Psi(1) \cdot \mathrm{d} \Psi(1) \cdot\left(e^{-\operatorname{ad}(X)} \cdot Y+U\right),
\end{aligned}
$$

where $\mathrm{d} \Psi(1)$ sends $\mathfrak{g}_{\mathbb{C}}$ onto $\mathfrak{q}_{\mathbb{C}}$. Note $\mathrm{P}_{\mathfrak{q}_{\mathbb{C}}}$ the projection of $\mathfrak{g}_{\mathbb{C}}$ onto $\mathfrak{q}_{\mathbb{C}}$ along $\mathfrak{h}_{\mathbb{C}}$, then

$$
\begin{aligned}
& \mathrm{P}_{\mathfrak{q}_{\mathbb{C}}}\left(e^{-\operatorname{ad}(X)} Y+U\right) \\
& =\frac{e^{-\operatorname{ad}(X)} Y+U-e^{\operatorname{ad}(X)} \sigma(Y)+U}{2} \\
& =\frac{e^{-\operatorname{ad}(X)} Y-e^{\operatorname{ad}(X)} \sigma(Y)}{2}+U \\
& =\operatorname{sh}(-\operatorname{ad}(X))\left(\frac{Y+\sigma(Y)}{2}\right)+\operatorname{ch}(\operatorname{ad}(X))\left(\frac{Y-\sigma(Y)}{2}\right)+U .
\end{aligned}
$$

Using the fact that $\sigma\left(\mathfrak{g}_{\beta}\right)=\mathfrak{g}_{-\beta}$, the Lie algebra $\mathfrak{g}$ can be written as

$$
\mathfrak{g}=\mathfrak{z} \oplus \sum_{\beta \in \triangle^{+}}(1+\sigma) \mathfrak{g}_{\beta} \oplus \mathfrak{a} \oplus \sum_{\beta \in \triangle^{+}}(1-\sigma) \mathfrak{g}_{\beta},
$$


where $\mathfrak{z}:=\operatorname{Lie}(Z)$. Then for all $Y \in \mathfrak{g}$ and $Y_{\beta} \in \mathfrak{g}_{\beta}$, $\mathrm{P}_{\mathfrak{q}_{\mathbb{C}}}\left(e^{-\operatorname{ad}(X)} Y+U\right)=\operatorname{sh}(-\beta(X))\left(Y_{\beta}+\sigma\left(Y_{\beta}\right)\right)+\operatorname{ch}(\beta(X))\left(Y_{\beta}-\sigma\left(Y_{\beta}\right)\right)+U$.

Let $\omega$ be the volume form on $\Xi$ which defines an invariant Haar measure on $\Xi$. Again, the volume form $\varphi^{*} \omega$ on $G / Z \times C^{+}$is given by

$$
\varphi^{*} \omega=c_{0} \prod_{\beta \in \triangle^{+}}(\operatorname{sh} 2 \beta(X))^{m_{\beta}} \omega_{1} \otimes \omega_{2},
$$

where $\omega_{1}$ is a volume form on $G / Z$ which defines an invariant measure, and $\omega_{2}$ is a volume form on $i \mathfrak{a}$ which defines a Lebesgue measure. Using the fact that $Z$ is compact, the integral formula holds.

\section{Weighted Bergman spaces and reproducing kernels.}

In this section we introduce the Bergman space $\mathcal{H}_{\nu}^{2}(\widetilde{\Xi})$ associated with the covering $\widetilde{\Xi}$. We establish a unitary isomorphism of $\mathcal{H}_{\nu}^{2}(\mathcal{D})$ on $\mathcal{H}_{\nu}^{2}(\widetilde{\Xi})$. Then we compute the explicit expression of the reproducing kernel of $\mathcal{H}_{\nu}^{2}(\widetilde{\Xi})$.

For a real $\nu$, let $\mathcal{O}_{\nu}(\widetilde{\Xi})$ be the space of holomorphic functions $F$ on $\widetilde{\Xi}$ which satisfy

$$
F(z, \zeta+2 \pi i)=e^{2 \pi i \frac{r}{2 r^{+}} \nu} F(z, \zeta)
$$

This condition will be called a monodromy condition. Remark that the function $\top_{\alpha}^{\frac{r}{4 n} \nu}$ belongs to $\mathcal{O}_{\nu}(\widetilde{\Xi})$.

For $\nu>\frac{2 n}{r}-1$, let $\mathcal{H}_{\nu}^{2}(\widetilde{\Xi})$ be the Hilbert space of functions $F \in \mathcal{O}_{\nu}(\widetilde{\Xi})$ such that

$$
\|F\|_{\nu}^{2}:=\int_{\Xi}|F(\widetilde{\xi})|^{2} p_{\nu}(\xi) d \xi<\infty
$$

where

$$
p_{\nu}(\xi)=\operatorname{det}(B(\xi, \bar{\xi}))^{\frac{r}{2 n} \nu-1}\left|\top_{\alpha}(\xi)\right|^{-\frac{r}{2 n} \nu+1},
$$

and $B(z, w)$ is the Bergman operator defined by $B(z, w):=\operatorname{id}_{\mathbb{V}_{\mathbb{C}}}-2 z \square w+$ $P(z) P(w)$.

Proposition 3.1. Let $z$ and $w$ be two invertible elements of $\mathbb{V}_{\mathbb{C}}$. Thus

$$
\operatorname{det}(B(z, w))=\Delta(z)^{\frac{2 n}{r}} \Delta\left(z^{-1}-w\right)^{\frac{2 n}{r}},
$$

where $\Delta$ is the determinant polynomial associated with $\mathbb{V}$.

Proof. By definition $\operatorname{det}(B(z, w))=\operatorname{det}\left(\operatorname{id}_{\mathbb{V}_{\mathbb{C}}}-2 z \square w+P(z) P(w)\right)$. According to [18] Proposition 4.13,

$$
z \square w=P\left(w^{-1}, z\right) P(w),
$$

where $P(z, w)=\frac{1}{2}(P(z+w)-P(z)-P(w))$. Then

$$
\operatorname{id}_{\mathbb{V}_{\mathbb{C}}}-2 z \square w+P(z) P(w)=\operatorname{id}_{\mathbb{V}_{\mathbb{C}}}-2 P\left(w^{-1}, z\right) P(w)+P(z) P(w) .
$$


Moreover

$$
\begin{aligned}
P\left(w^{-1}-z\right) & =P(w)^{-1}+2 P\left(w^{-1},-z\right)+P(z) \\
& =P\left(w^{-1}\right)-2 P\left(w^{-1}, z\right)+P(z) .
\end{aligned}
$$

Hence we deduce the following equalities

$$
-2 P\left(w^{-1}, z\right) P(w)=P\left(w^{-1}-z\right) P(w)-P(z) P(w)-\operatorname{id}_{V_{\mathbb{C}}},
$$

and

$$
\operatorname{id}_{\mathbb{C}}-2 z \square w+P(z) P(w)=P\left(w^{-1}-z\right) P(w) .
$$

Then, we have

$$
\operatorname{det}\left(\operatorname{id}_{\mathbb{V}_{\mathbb{C}}}-2 z \square w+P(z) P(w)\right)=\operatorname{det}\left(P\left(w^{-1}-z\right)\right) \operatorname{det}(P(w)),
$$

where $\operatorname{det}(P(w))=\Delta(w)^{\frac{2 n}{r}}$. Finally

$$
\operatorname{det}(B(z, w))=\Delta(w)^{\frac{2 n}{r}} \Delta\left(w^{-1}-z\right)^{\frac{2 n}{r}}=\Delta(z)^{\frac{2 n}{r}} \Delta\left(z^{-1}-w\right)^{\frac{2 n}{r}} .
$$

The universal covering $\widetilde{G}$ of $G$ can be realized as the set of pairs $(g, \varphi)$ with $g \in G$ and $\varphi$ a holomorphic function on $\mathcal{D}$ defined by

$$
e^{\varphi(z)}=\operatorname{det}(D g(z)),
$$

where $D g(z)$ is the differential of the map $z \mapsto g \cdot z$. The product on $\widetilde{G}$ is given by

$$
\left(g_{1}, \varphi_{1}\right) \cdot\left(g_{2}, \varphi_{2}\right)=\left(g_{1} g_{2}, \varphi_{3}\right)
$$

where $\varphi_{3}(z)=\varphi_{1}\left(g_{2} \cdot z\right)+\varphi_{2}(z)$. For $\widetilde{g}=(g, \varphi) \in \widetilde{G}$, and $\kappa \in \mathbb{R}$, we will write

$$
\operatorname{det}(D g(z))^{\kappa}:=e^{\kappa \varphi(z)} .
$$

Let $\widetilde{\Gamma(C)}:=\widetilde{G} \widetilde{\exp }(C)$ be the semigroup associated with the covering $\widetilde{G}$ where $\widetilde{\exp }: \mathfrak{g} \rightarrow \widetilde{G}$. We denote by $\widetilde{\Gamma\left(C^{0}\right)}$ the interior of $\widetilde{\Gamma(C)}$. The linear action of $\widetilde{G}$ on the space $\mathcal{H}_{\nu}^{2}(\widetilde{\Xi})$ is given by

$$
\left(\pi_{0}(\widetilde{g}) F\right)(\widetilde{\xi})=F(g \cdot \widetilde{\xi}), \quad \widetilde{g}^{-1}=(g, \varphi),
$$

where $g \cdot \widetilde{\xi}=\left(g \cdot \xi, \zeta^{\prime}\right)$ and $e^{\frac{2 n}{r^{+}} \zeta^{\prime}}=\operatorname{det}(P(g \cdot \xi+\alpha(g \cdot \xi)))($ cf. [19] Lemma 5.1).

The representation $\pi_{0}$ extends to a continuous representation of $\widetilde{\Gamma(-C)}$ and a holomorphic one of $\widetilde{\Gamma\left(-C^{0}\right)}($ cf. $[25])$.

We recall that the Bergman space $\mathcal{H}_{\nu}^{2}(\mathcal{D})$ is the Hilbert space of holomorphic functions $f$ on $\mathcal{D}$ such that

$$
\|f\|^{2}=\int_{\mathcal{D}}|f(z)|^{2} \operatorname{det}(B(z, \bar{z}))^{\frac{r}{2 n} \nu-1} d \lambda(z)<\infty,
$$


where $\lambda$ denote the Lebesgue measure (cf. [12]). The action of $\widetilde{G}$ on $\mathcal{H}_{\nu}^{2}(\mathcal{D})$ is given by

$$
\left(\pi_{\nu}(\widetilde{g}) f\right)(z)=e^{\frac{r}{2 n} \nu \varphi(z)} f(g \cdot z), \quad \widetilde{g}^{-1}=(g, \varphi) .
$$

The unitary representation $\pi_{\nu}$ extends to a continuous representation of $\widetilde{\Gamma(-C)}$ and a holomorphic one of $\widetilde{\Gamma\left(-C^{0}\right)}$.

Let $\mathcal{A}_{\nu}$ be the operator given by

$$
\begin{aligned}
\mathcal{A}_{\nu}: \mathcal{H}_{\nu}^{2}(\mathcal{D}) & \longrightarrow \mathcal{O}_{\nu}(\widetilde{\Xi}), \\
f & \mapsto \mathcal{A}_{\nu}(f)=\top_{\alpha} \alpha^{\frac{r}{4 n} \nu} f .
\end{aligned}
$$

Since $\top_{\alpha^{\frac{r}{4 n}} \nu} \in \mathcal{O}_{\nu}(\widetilde{\Xi})$, the operator $\mathcal{A}_{\nu}$ is well defined.

Theorem 3.1. The operator $\mathcal{A}_{\nu}$ is a unitary isomorphism of $\mathcal{H}_{\nu}^{2}(\mathcal{D})$ onto $\mathcal{H}_{\nu}^{2}(\widetilde{\Xi})$ intertwining the representations $\pi_{\nu}$ and $\pi_{0}$.

Proof. Since $\Sigma$ is an analytic set of measure zero and $d \xi=\frac{d \lambda(z)}{\left|\top_{\alpha}(z)\right|}$,

$$
\begin{aligned}
\left\|\mathcal{A}_{\nu}(f)\right\|_{\nu}^{2} & =\int_{\Xi}\left|\mathcal{A}_{\nu}(f)(\widetilde{\xi})\right|^{2} p_{\nu}(\xi) d \xi \\
& =\int_{\Xi}|f(\xi)|^{2}\left|\top_{\alpha}(\widetilde{\xi})\right|^{\frac{r}{2 n} \nu} \operatorname{det}(B(\xi, \bar{\xi}))^{\frac{r}{2 n} \nu-1} \frac{d \xi}{\left|\top_{\alpha}(\widetilde{\xi})\right|^{\frac{r}{2 n}} \nu-1} \\
& =\int_{\mathcal{D}}|f(z)|^{2} \operatorname{det}(B(z, \bar{z}))^{\frac{r}{2 n} \nu-1} d \lambda(z)=\|f\|^{2} .
\end{aligned}
$$

If $f$ belongs to $\mathcal{H}_{\nu}^{2}(\mathcal{D})$ then $\mathcal{A}_{\nu}(f) \in \mathcal{O}_{\nu}(\widetilde{\Xi})$ and

$$
\int_{\Xi}\left|\mathcal{A}_{\nu} f(\widetilde{\xi})\right|^{2} p_{\nu}(\xi) d \xi<\infty .
$$

Hence the image of $\mathcal{A}_{\nu}$ is containd in $\mathcal{H}_{\nu}^{2}(\widetilde{\Xi})$ and $\mathcal{A}_{\nu}$ is isometric.

Moreover $\mathcal{A}_{\nu}$ is surjective. In fact, let $F \in \mathcal{H}_{\nu}^{2}(\widetilde{\Xi})$, then in particular $F \in \mathcal{O}_{\nu}(\widetilde{\Xi})$. Since $\operatorname{det}(P(\widetilde{z}+\alpha \widetilde{z}))^{-\frac{r}{4 n} \nu} \in \mathcal{O}_{-\nu}(\widetilde{\Xi})$, the function

$$
f(z):=\top_{\alpha}(\widetilde{z})^{-\frac{r}{4 n} \nu} F(\widetilde{z}), \quad \widetilde{z}=(z, \zeta) \in \widetilde{\Xi},
$$

is holomorphic on $\Xi=\mathcal{D} \backslash \Sigma$. Moreover, the function $f$ belongs to $\mathcal{H}_{\nu}^{2}(\mathcal{D})$. In fact

$$
\begin{aligned}
\|f\|^{2} & =\int_{\mathcal{D}}|F(\widetilde{z})|^{2}\left|\top_{\alpha}(z)\right|^{-\frac{r}{2 n} \nu} \operatorname{det}(B(z, \bar{z}))^{\frac{r}{2 n} \nu-1} d \lambda(z) \\
& =\int_{\Xi}|F(\widetilde{\xi})|^{2}\left|\top_{\alpha}(\xi)\right|^{-\frac{r}{2 n} \nu+1} \operatorname{det}(B(\xi, \bar{\xi}))^{\frac{r}{2 n} \nu-1} d \xi=\|F\|_{\nu}^{2}<\infty .
\end{aligned}
$$

Then $f$ is a holomorphic function on $\mathcal{D} \backslash \Sigma$ and belongs to $L_{\nu}^{2}(\mathcal{D})$. Hence $f$ extends to a holomorphic function on $\mathcal{D}$. This is the content of the following lemma. 
Lemma 3.2 (cf. [6], [27]). Let $U$ be a domain in $\mathbb{C}^{n}$ and let $A$ be an analytic set such that $\operatorname{codim}_{\mathbb{R}}(A) \geq 1$. If $f \in \mathcal{O}(U \backslash A)$ and if $f \in L^{2}(U)$, then $f$ extends to a holomorphic function on $U$.

It remains to show that $\mathcal{A}_{\nu}$ intertwining the representations $\pi_{0}$ and $\pi_{\nu}$. In fact it follows from $[3] 1.3(9)$ that $\top_{\alpha}(g \cdot \widetilde{\xi})^{\frac{r}{4 n} \nu}=e^{\frac{r}{2 n} \nu \varphi(\xi)} \top_{\alpha}(\widetilde{\xi})^{\frac{r}{4 n} \nu}$. Hence

$$
\begin{aligned}
\mathcal{A}_{\nu}\left(\pi_{\nu}(\widetilde{g}) f\right)(\widetilde{\xi}) & =\top_{\alpha}(\widetilde{\xi})^{\frac{r}{4 n} \nu} e^{\frac{r}{2 n} \nu \varphi(\xi)} f(g \cdot \xi) \quad\left(\widetilde{g}^{-1}=(g, \varphi)\right) \\
& =e^{-\frac{r}{2 n} \nu \varphi(\xi)} \top_{\alpha}(g \cdot \widetilde{\xi})^{\frac{r}{4 n} \nu} e^{\frac{r}{2 n} \nu \varphi(\xi)} f(g \cdot \xi) \\
& =\left(\pi_{0}(\widetilde{g}) \mathcal{A}_{\nu} f\right)(\widetilde{\xi}) .
\end{aligned}
$$

Proposition 3.3. The reproducing kernel of the Bergman space $\mathcal{H}_{\nu}^{2}(\widetilde{\Xi})$ is equal to

$$
K_{\nu}\left(\widetilde{\xi}_{1}, \widetilde{\xi}_{2}\right)=c_{\nu} \top_{\alpha}\left(\widetilde{\xi}_{1}\right)^{\frac{r}{4 n} \nu} \operatorname{det}\left(B\left(\xi_{1}, \bar{\xi}_{2}\right)\right)^{-\frac{r}{2 n} \nu} \overline{T_{\alpha}\left(\widetilde{\xi}_{2}\right)}{ }^{\frac{r}{4 n} \nu},
$$

where $c_{\nu}$ is the positive constant

$$
c_{\nu}=\frac{1}{\pi^{n}} \prod_{j=1}^{r} \frac{\Gamma\left(\nu-(j-1) \frac{n-r}{r(r-1)}\right)}{\Gamma\left(\nu-\frac{n}{r}-(j-1) \frac{n-r}{r(r-1)}\right)} .
$$

The definition of $\bar{\top}_{\alpha}\left(\widetilde{\xi}_{2}\right)^{\frac{r}{4 n}} \nu$ is similar to that given on (3).

Proof. The reproducing kernel of $\mathcal{H}_{\nu}^{2}(\mathcal{D})$ is given by

$$
K_{\nu}^{\mathcal{D}}\left(z, z^{\prime}\right)=c_{\nu} \operatorname{det}\left(B\left(z, \bar{z}^{\prime}\right)\right)^{-\frac{r}{2 n} \nu} .
$$

From the definition of $\mathcal{A}_{\nu}$, the reproducing kernel of $\mathcal{H}_{\nu}^{2}(\widetilde{\Xi})$ is equal to

$$
\begin{aligned}
K_{\nu}\left(\widetilde{\xi}, \widetilde{\xi}^{\prime}\right) & =\top_{\alpha}(\widetilde{\xi})^{\frac{r}{4 n} \nu} K_{\nu}^{\mathcal{D}}\left(\xi, \xi^{\prime}\right) \overline{T_{\alpha}\left(\widetilde{\xi}^{\prime}\right)^{\frac{r}{4 n}} \nu} \\
& =c_{\nu} \top_{\alpha}(\widetilde{\xi})^{\frac{r}{4 n} \nu} \operatorname{det}\left(B\left(\xi, \overline{\xi^{\prime}}\right)\right)^{-\frac{r}{2 n} \nu} \overline{\top_{\alpha}\left(\widetilde{\xi}^{\prime}\right)^{\frac{r}{4 n}} \nu} .
\end{aligned}
$$

\section{Holomorphic discrete series of $\widetilde{G}$.}

Recall that $\left\{e_{1}, \ldots, e_{r^{+}}\right\}$is the Jordan frame of $R^{+}$and $\mathfrak{k} \cap \mathfrak{q}=\{(0, i L(v), 0) \mid$ $\left.v \in \mathbb{V}^{+}\right\}$.

Let $\mathfrak{a}$ be the Cartan subalgebra in $\mathfrak{k} \cap \mathfrak{q}$ defined by

$$
\mathfrak{a}=\left\{\left(0, i \sum_{j=1}^{r^{+}} t_{j} L\left(e_{j}\right), 0\right) \mid t_{j} \in \mathbb{R}\right\} .
$$


We denote by $\triangle$ the root system $\triangle\left(\mathfrak{g}_{\mathbb{C}}, i \mathfrak{a}\right), \Delta^{+}$the positive system with respect to the positive Weyl chamber $(i \mathfrak{a})^{+}$defined by

$$
(i \mathfrak{a})^{+}=\left\{\left(0, \sum_{j=1}^{r^{+}} t_{j} L\left(e_{j}\right), 0\right) \mid 0<t_{1}<\cdots<t_{r^{+}}\right\} .
$$

Let $X_{0}:=(0, I, 0) \in \mathfrak{g}_{\mathbb{C}}$. The eigenvalues of $\operatorname{ad}\left(X_{0}\right)$ are 1,0 , and -1 . Let $\triangle_{0}:=\left\{\alpha \in \triangle^{+} \mid \alpha\left(X_{0}\right)=0\right\}$ and $\triangle_{1}:=\left\{\alpha \in \triangle^{+} \mid \alpha\left(X_{0}\right)=1\right\}$. Then $\triangle^{+}=\triangle_{0} \cup \triangle_{1}$. The roots belonging to $\triangle_{0}$ are called compact and the roots belonging to $\triangle_{1}$ noncompact. Let $\rho:=\frac{1}{2} \sum_{\alpha \in \Delta^{+}} m_{\alpha} \alpha$ be one half of the positive roots weighted by the dimension $m_{\alpha}$ of the root spaces. For the description and computation of $\rho$ we refer to [4]. See also [8].

Let $\pi$ be a unitary representation of the Lie group $G$ on a Hilbert space $\mathcal{H}$, and let $C$ be an invariant and regular cone in $i \mathfrak{g}$. The representation $\pi$ is called $C$-positive if for all $X \in C$ and for all $\mathcal{C}^{\infty}$ vector $v$,

$$
\frac{\mathrm{d}}{\mathrm{d} \mathrm{t} \mid \mathrm{t}=0}\langle\pi(\exp (t X)) v \mid v\rangle \leq 0 \text {. }
$$

Let $\mathcal{R}$ be the set of the weights $\mu=\left(\mu_{1}, \mu_{2}, \ldots, \mu_{r^{+}}\right) \in \mathbb{R}^{r^{+}}$such that

$$
\mu_{i}-\mu_{i+1} \in \mathbb{N}, \quad 1 \leq i \leq r^{+}-1 .
$$

(If $\mathbb{V}^{+}$is a direct sum of two simple algebras with ranks $p$ and $q$ such that $p+q=r^{+}(=r)$, then $i \neq p$. $)$

For $\mu \in \mathcal{R}$ and $\beta$ a noncompact positive root. By [14], the "HarishChandra" condition $\langle\rho-\mu, \beta\rangle \leq 0$ can be written as

(*) $\mathbb{V}^{+}$is simple $\quad \mu_{r^{+}}>\frac{n}{2 r}-\frac{d+1}{8} \quad$ if $\quad r=r^{+},\left(d:=\frac{2(n-r)}{r(r-1)}\right)$

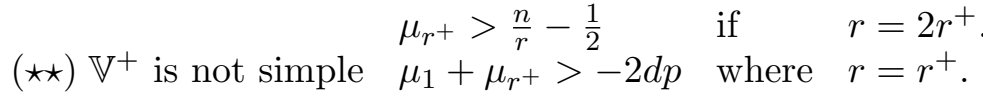

If $\mu \in \mathcal{R}$ and satisfies the "Harish-Chandra" condition, then we can associate to $\mu$ a unitary and $C$-positive representation $\left(\pi_{\mu}, \mathcal{W}_{\mu}\right)$ of $\widetilde{G}$ with highest weight $\mu$. This representation extends to a continuous representation of $\widetilde{\Gamma(C)}$ which is holomorphic on $\widetilde{\Gamma\left(C^{0}\right)}$.

Let $A:=\exp \mathfrak{a}$, and $\mathfrak{g}_{+}^{\mathbb{C}}:=\sum_{\beta \in \triangle^{+}} \mathfrak{g}_{\beta}^{\mathbb{C}}$.

Definition 4.1. A holomorphic function $\Phi$ in $\widetilde{\Xi}$ will be called a conical function if there exists a continuous character $\chi_{\mu}$ of $A$ such that

$$
\begin{aligned}
\mathcal{I}(a) \Phi & =\chi_{\mu}(a) \Phi, \quad(a \in A), \\
d \mathcal{I}(X) \Phi & =0, \quad\left(X \in \mathfrak{g}_{+}^{\mathbb{C}},\right)
\end{aligned}
$$

where $(\mathcal{I}(g) F)(\widetilde{\xi})=F\left(g^{-1} \cdot \widetilde{\xi}\right)$. 
For all $\mathbf{s}=\left(s_{1}, s_{2}, \ldots, s_{r}\right) \in \mathbb{C}^{r}$ and $z$ in $\mathbb{V}_{\mathbb{C}}$, we write

$$
\Delta_{\mathbf{S}}(z):=\Delta_{1}(z)^{s_{1}-s_{2}} \Delta_{2}(z)^{s_{2}-s_{3}} \ldots \Delta_{r}(z)^{s_{r}},
$$

where $\Delta_{j}$ is the principal minor of order $j$ (cf. [12]).

For $\mu=\left(\mu_{1}, \mu_{2}, \ldots, \mu_{r^{+}}\right)$, let,

$$
\Phi_{\mu}(\widetilde{z}):=\Delta_{\mu}\left(\frac{\widetilde{z}+\alpha(\widetilde{z})}{2}\right) .
$$

The function $\Phi_{\mu}$ satisfies the monodromy condition.

Proposition 4.2. The function $\Phi_{\mu}$ is conical, and any conical function is proportional to $\Phi_{\mu}$.

The proof is similar to that given for Proposition XI.2.1 in [12].

Note that $\mathcal{W}_{\mu}^{\infty}$ (resp. $\left.\mathcal{W}_{\mu}^{-\infty}\right)$ is the vector space of $\mathcal{C}^{\infty}$ (resp. distribution) vectors of $\mathcal{W}_{\mu}$, and $\left(\mathcal{W}_{\mu}^{-\infty}\right)^{H}$ the vector space of $H$-invariant distribution vectors of $\mathcal{W}_{\mu}$. Let $\mathcal{R}_{H}$ be the subset of highest weight $\mu \in \mathcal{R}$ such that $\left(\mathcal{W}_{\mu}^{-\infty}\right)^{H} \neq\{0\}$.

For $\mu \in \mathcal{R}_{H}$, we denote $\psi_{\mu}$ an $H$-invariant distribution vector. For all element $w \in \mathcal{W}_{\mu}$, the holomorphic mapping $\mathcal{F}: \mathcal{W}_{\mu} \longrightarrow \mathcal{O}(\widetilde{\Xi}), \quad w \mapsto$ $\mathcal{F}(w)(\widetilde{\xi}):=\left\langle\pi_{\mu}\left(\widetilde{\gamma}_{1}^{-1}\right) w \mid \psi_{\mu}\right\rangle$ where $\widetilde{\xi}=\widetilde{\gamma} \cdot H$, is a continuous embedding. Then the representation $\pi_{\mu}$ is realized on a Hilbert space $\mathcal{H}_{\mu}$ of holomorphic functions on $\widetilde{\Xi}$. In the case where $w=v_{\mu}$, a normalized highest weight vector, we denote $\mathcal{F}_{\mu}(\widetilde{\xi}):=\left\langle\pi_{\mu}\left(\widetilde{\gamma}^{-1}\right) v_{\mu} \mid \psi_{\mu}\right\rangle$. The function $\mathcal{F}_{\mu}$ is a conical function.

Let $\mathcal{R}_{\nu, H}$ be the highest weight subset of $\mathcal{R}_{H}$ such that $\mathcal{F}_{\mu} \in \mathcal{O}_{\nu}(\widetilde{\Xi})$.

Proposition 4.3. The function $\mathcal{F}_{\mu}$ satisfies the monodromy condition, i.e., $\mathcal{F}_{\mu} \in \mathcal{O}_{\nu}(\widetilde{\Xi})$, if and only if

$$
\mu_{i} \in \mathbb{Z}+\frac{\nu}{2}, \quad\left(1 \leq i \leq r^{+}\right) \quad \text { if } r=r^{+}
$$

and

$$
\mu_{i} \in \mathbb{Z}+\nu, \quad\left(1 \leq i \leq r^{+}\right) \quad \text { if } r=2 r^{+} .
$$

Proof. Since $\mathcal{F}_{\mu}$ is conical, then it is proportional to $\Phi_{\mu}$. If $\mathbb{V}^{+}$is simple, then $T_{\alpha}$ is proportional to the Jordan determinant $\Delta$ of $\mathbb{V}^{+}$. In fact, $T_{\alpha}$ is homogeneous of degree $2 n$ and $\Delta$ is homogeneous of degree $r^{+}$, then $\top_{\alpha}(\widetilde{z})=\Delta(\widetilde{z}+\alpha(\widetilde{z}))^{\frac{2 n}{r^{+}}}$and $\mathcal{F}_{\mu} \in \mathcal{O}_{\nu}(\widetilde{\Xi})$ if and only if $\mu_{r^{+}} \in \mathbb{Z}+\frac{r}{2 r^{+}} \nu$. Using the fact that $\mu_{i}-\mu_{i+1} \in \mathbb{N}$, the result holds for $\mathbb{V}^{+}$simple.

If $\mathbb{V}^{+}$is a direct sum of two simple Jordan algebras $\mathbb{V}_{1}^{+}$of rank $p$ and $\mathbb{V}_{2}^{+}$ of rank $q$ such that $p+q=r^{+}(=r)$, then there exist $z_{1} \in \mathbb{V}_{1, \mathbb{C}}^{+}$and $z_{2} \in \mathbb{V}_{2, \mathbb{C}}^{+}$ such that $z+\alpha(z)=z_{1}+z_{2}$ and $\top_{\alpha}(\widetilde{z})=\Delta^{(1)}\left(\widetilde{z}_{1}\right)^{\frac{2 n}{r}} \Delta^{(2)}\left(\widetilde{z}_{2}\right)^{\frac{2 n}{r}}$ where $\Delta^{(1)}$ (resp. $\left.\Delta^{(2)}\right)$ is the Jordan determinant of $\mathbb{V}_{1}^{+}\left(\right.$resp. $\left.\mathbb{V}_{2}^{+}\right)$. Hence $\mathcal{F}_{\mu} \in \mathcal{O}_{\nu}(\widetilde{\Xi})$ 
if and only if $\mu_{p} \in \mathbb{Z}+\frac{\nu}{2}$ and $\mu_{r} \in \mathbb{Z}+\frac{\nu}{2}$. The assertion follows from the fact that $\mu_{i}-\mu_{i+1} \in \mathbb{N}$ for all $i \neq p$.

Remark. In [1] we consider the case $G / H \simeq U(p, q)$ and we establish another isomorphism between $\mathcal{H}_{\nu}^{2}(\mathcal{D})$ and $\mathcal{H}_{\nu}^{2}(\widetilde{\Xi})$. The correspondence between the present isomorphism $\mathcal{A}_{\nu}$ and the one used in [1] is given by $f \mapsto \operatorname{det}(A)^{\frac{\nu}{2}} \operatorname{det}(D)^{-\frac{\nu}{2}} f(z)$ for all $z=\left[\begin{array}{ll}A & B \\ C & D\end{array}\right] \in \mathcal{D}$. This correspondence explains the shift between the highest weight $\mu$ shown in $[\mathbf{1}]$ and the present form of $\mu$.

Since $\nu$ is very large $\left(\nu>\frac{2 n}{r}-1\right)$, the representation $\pi_{\mu}$ satisfies the Harish-Chandra condition for all $\mu \in \mathcal{R}_{\nu, H}$.

Let

$$
C_{\mu}(\nu)=\int_{\Xi}\left|\left\langle\pi_{\mu}\left(\widetilde{\gamma}^{-1}\right) v_{\mu} \mid \psi_{\mu}\right\rangle\right|^{2} p_{\nu}(\xi) d \xi
$$

Proposition 4.4. For $\mu \in \mathcal{R}_{\nu, H}$, the Hilbert space $\mathcal{H}_{\mu}$ belongs to $\mathcal{H}_{\nu}^{2}(\widetilde{\Xi})$ if and only if $C_{\mu}(\nu)$ is finite.

In this case we denote $\mu \in \mathcal{R}_{\nu, H}^{\prime}$.

Proof. This is proved in [1] Proposition 4.2.

Hence the Plancherel Theorem can be written as:

Theorem 4.1 (cf. [13]). The Bergman space $\mathcal{H}_{\nu}^{2}(\widetilde{\Xi})$ is decomposed multiplicity-free and discretely into irreducible Hilbert subspaces,

$$
\mathcal{H}_{\nu}^{2}(\widetilde{\Xi})=\bigoplus_{\mu \in \mathcal{R}_{\nu, H}^{\prime}} \mathcal{H}_{\mu}
$$

Moreover, the reproducing kernel can be written as

$$
K_{\nu}\left(\widetilde{\xi}_{1}, \widetilde{\xi}_{2}\right)=\sum_{\mu \in \mathcal{R}_{\nu, H}^{\prime}} \frac{1}{C_{\mu}(\nu)}\left\langle\pi_{\mu}\left(\widetilde{\gamma}_{2}^{\sharp} \widetilde{\gamma}_{1}\right)^{-1} \psi_{\mu} \mid \psi_{\mu}\right\rangle .
$$

The series converges uniformly on compact subsets of $\widetilde{\Xi} \times \widetilde{\Xi}$.

\section{Computation of the constant $C_{\mu}(\nu)$.}

Let $\mathcal{M}$ be a differentiable manifold. A causal structure on $\mathcal{M}$ is a field of cones $\mathcal{M} \ni x \mapsto C_{x} \subset T_{x} \mathcal{M}$. The cones $C_{x}$ are assumed to be closed, convex, proper, and with nonempty interior. Furthermore the cones $C_{x}$ depend smoothly on $x$. A piecewise $\mathcal{C}^{1}$ curve $\gamma:[0,1] \longrightarrow \mathcal{M}$ is said to be causal if for all $t$, the derivative $\dot{\gamma}(t)$ belongs to the cone $C_{\gamma(t)}$. The causal structure is said to be global if there exists no nontrivial closed causal curve. 
In that case one defines a partial ordering on $\mathcal{M}$ in the following way: One writes $x \leq y$ if there exists a causal curve from $x$ to $y$.

Let $\mathfrak{g}$ be the Lie algebra defined in Section 2. Let $\sigma$ be an involutive automorphism of $\mathfrak{g}$ that commutes with the Cartan involution $\theta$ where $\mathfrak{g}=$ $\mathfrak{k} \bigoplus^{\theta} \mathfrak{p}$, and $\mathfrak{g}=\mathfrak{h} \bigoplus^{\sigma} \mathfrak{q}$.

Let $G^{c}:=\left(\underline{G}^{(\alpha)}\right)_{0}$ where $\underline{G}^{(\alpha)}=\{g \in \underline{G} \mid \alpha \circ g \circ \alpha=g\}$ and the subscript 0 means the identity component. The group $G^{c}$ is the group of holomorphic automorphisms of the tube domain $T_{\Omega^{+}}$associated with the involution $\alpha$ defined by

$$
T_{\Omega^{+}}:=\mathbb{V}^{-}+\Omega^{+}=\left\{x+y \mid x \in \mathbb{V}^{-}, \quad y \in \Omega^{+}\right\}
$$

where $\Omega^{+}:=\mathbb{V}^{+} \cap \Omega$ and $\Omega$ is the symmetric cone associated with $\mathbb{V}$. (If $\mathbb{V}$ and $\mathbb{V}^{+}$are simple, the cone $\Omega^{+}$coincides with the open cone associated with the Jordan algebra $\mathbb{V}^{+}$.) The group $G^{c}$ is the $c$-dual group of $G$. We consider on $\mathcal{M}:=G^{c} / H$ the causal structure defined by the field of cones

$$
C_{x}=-\bar{\Omega} .
$$

The noncompactly causal symmetric space $\mathcal{M}$ is an ordered symmetric space. By [3], the intersection $\mathcal{M} \cap \mathbb{V}$ is a union of connected components of the set $\left\{x \in \mathbb{V} \mid \operatorname{det} B_{\alpha}(x, x) \neq 0\right\}$ where $B_{\alpha}(x, y):=B(x, \alpha y)$ and $B(x, y)$ is the Bergman operator. In particular

$$
(\mathcal{M} \cap \mathbb{V})_{0}=\left\{x \in \mathbb{V} \mid \operatorname{det} B_{\alpha}(x, x) \neq 0\right\}_{0} .
$$

Let $\mathfrak{g}^{c}=\operatorname{Lie}\left(G^{c}\right)$ (the $c$-dual algebra of $\mathfrak{g}$ ). We denote also by $\sigma$ the $\mathbb{C}$-linear extention of $\sigma$ to the complexified algebra $\mathfrak{g}_{\mathbb{C}}$ of $\mathfrak{g}$. The involution $\theta^{c}:=\theta \sigma_{\mid \mathfrak{g}^{c}}$ is a Cartan involution of $\mathfrak{g}^{c}$. Let $\mathfrak{a}$ be a maximal abelian subspace in $\mathfrak{p}^{c} \cap i \mathfrak{q}$ where $\mathfrak{p}^{c}:=\left(\mathfrak{g}^{c}\right)^{\theta^{c}}$ (note that $i \mathfrak{a}$ is a maximal abelian subspace in $\mathfrak{k} \cap \mathfrak{q}), \triangle$ the root system for the pair $\left(\mathfrak{g}^{c}, \mathfrak{a}\right)$, and let $\triangle^{+}$be the positive root system with respect to the positive Weyl chamber $\mathfrak{a}^{+}$(see (5)).

Let

$$
\begin{gathered}
\mathfrak{n}:=\bigoplus_{\beta \in \triangle^{+}} \mathfrak{g}_{\beta}^{c}, \quad \overline{\mathfrak{n}}:=\bigoplus_{\beta \in-\triangle^{+}} \mathfrak{g}_{\beta}^{c}, \\
N:=\exp \mathfrak{n}, \quad \bar{N}:=\exp \overline{\mathfrak{n}}, \quad A:=\exp \mathfrak{a} .
\end{gathered}
$$

Let $x_{0}:=e H$ be the base point of $G^{c} / H$. The map

$$
N \times A \longrightarrow \mathcal{M}, \quad(n, a) \mapsto n a \cdot x_{0},
$$

is a diffeomorphism of $N \times A$ onto its open image $N A \cdot x_{0}$. For all $x=$ $n \exp (X) \cdot x_{0}(X \in \mathfrak{a})$, we write $X=A(x)$. We denote $a_{H}(x):=\exp A(x)$.

Let $\mathcal{M}^{+}$be the subset of $\mathcal{M}$ defined by

$$
\mathcal{M}^{+}:=\left\{x \in \mathcal{M} \mid x \geq x_{0}\right\},
$$

called the future of $x_{0}$. By $[\mathbf{9}], \mathcal{M}^{+} \subset N A \cdot x_{0}$. 
The spherical Laplace transform of an $H$-invariant function $f$ is defined by

$$
\widehat{f}(\lambda)=\int_{\mathcal{M}^{+}} f(x) a_{H}(x)^{-\lambda} d x, \quad \lambda \in \mathfrak{a}_{\mathbb{C}}^{*}
$$

Using the following integral formula,

$$
\int_{\mathcal{M}^{+}} f(x) d x=\int_{-\mathfrak{a}^{+}} \int_{H} f\left(h \exp (X) \cdot x_{0}\right) d h \prod_{\beta \in-\triangle^{+}}(\operatorname{sh}\langle\beta, X\rangle)^{m_{\beta}} d X
$$

the spherical Laplace transform can be written as

$$
\widehat{f}(\lambda)=c \int_{-\mathfrak{a}^{+}} f\left(\exp (X) \cdot x_{0}\right) \varphi_{\lambda}(\exp (X)) \prod_{\beta \in-\triangle^{+}}(\operatorname{sh}\langle\beta, X\rangle)^{m_{\beta}} d X,
$$

where $\varphi_{\lambda}$ is the spherical function of the ordered symmetric space $\mathcal{M}$, defined in the interior $S^{0}$ of $S:=\left\{g \in G^{c} \mid g \cdot x_{0} \geq x_{0}\right\} \subset N A H$,

$$
\varphi_{\lambda}(g)=\int_{H} a_{H}(h g)^{-\lambda} d h
$$

(cf. [10]). The $c$-function of the symmetric space $\mathcal{M}$, which we denote by $c_{\mathcal{M}}$, is defined by the integral

$$
c_{\mathcal{M}}(\lambda)=\int_{\bar{N} \cap H A N} a_{H}(\bar{n})^{-(\lambda+\rho)} d \bar{n} .
$$

Remark. From Theorem 2.3 and the integral formula (6), we obtain

$$
\int_{\Xi} f(\xi) d \xi=c_{0} \int_{G / H} \int_{\mathcal{M}^{+}} f\left(g \cdot i x^{\frac{1}{2}}\right) d \dot{g} d x .
$$

This integral formula is a generalization of that given in Proposition X.3.4 of $[\mathbf{1 2}]$ where $G=\underline{K}$.

Let $\mathcal{W}_{\mu}^{\omega}$ (resp. $\left.\mathcal{W}_{\mu}^{-\omega}\right)$ be the space of analytic (resp. hyperfunction) vectors of $\mathcal{W}_{\mu}$. By [5] Theorem 1.1, $\left(\mathcal{W}_{\mu}^{-\omega}\right)^{H}=\left(\mathcal{W}_{\mu}^{-\infty}\right)^{H}$ where $\left(\mathcal{W}_{\mu}^{-\omega}\right)^{H}$ is the subspace of $H$-invariant hyperfunction vectors of $\mathcal{W}_{\mu}^{-\omega}$. Moreover, if the representation $\left(\pi_{\mu}, \mathcal{W}_{\mu}\right)$ satisfies the Harish-Chandra condition, the linear form

$$
L_{\mu}(f)=\int_{H}\left\langle\pi_{\mu}(h) f \mid v_{\mu}\right\rangle d h, \quad f \in \mathcal{W}_{\mu}^{\omega}
$$

defines an $H$-invariant hyperfunction vector (cf. [20]). Using the fact that $\operatorname{dim}\left(\mathcal{W}_{\mu}^{-\infty}\right)^{H} \leq 1$, and we deduce that if $\psi_{\mu}$ is an $H$-invariant distribution vector, there exist a constant $c_{0}$ such that

$$
\left\langle f \mid \psi_{\mu}\right\rangle=c_{0} L_{\mu}(f), \quad f \in \mathcal{W}_{\mu}^{\infty} .
$$

In particular if $f=v_{\mu}$ then

$$
\left\langle v_{\mu} \mid \psi_{\mu}\right\rangle=c_{0} \int_{H}\left\langle\pi_{\mu}(h) v_{\mu} \mid v_{\mu}\right\rangle d h .
$$


Using the integral formula for all functions $f \in L^{1}(H)$,

$$
\int_{H} f(h) d h=\int_{\bar{N} \cap H A N} f(h(\bar{n})) a_{H}(\bar{n})^{-2 \rho} d \bar{n},
$$

(cf. [24]), we deduce that

$$
\begin{aligned}
L_{\mu}\left(v_{\mu}\right) & =\int_{\bar{N} \cap H A N}\left\langle\pi_{\mu}(h(\bar{n})) v_{\mu} \mid v_{\mu}\right\rangle a_{H}(\bar{n})^{-2 \rho} d \bar{n} \\
& =\int_{\bar{N} \cap H A N}\left\langle\pi_{\mu}\left(a_{H}(\bar{n})^{-1} \bar{n}\right) v_{\mu} \mid v_{\mu}\right\rangle a_{H}(\bar{n})^{-2 \rho} d \bar{n} \quad\left(\pi_{\mu}(n(\bar{n})) v_{\mu}=v_{\mu}\right) \\
& =\int_{\bar{N} \cap H A N}\left\langle\pi_{\mu}(\bar{n}) v_{\mu} \mid v_{\mu}\right\rangle a_{H}(\bar{n})^{-2 \rho-\mu} d \bar{n} \\
& =\int_{\bar{N} \cap H A N}\left\langle v_{\mu} \mid \pi_{\mu}(\bar{n})^{*} v_{\mu}\right\rangle a_{H}(\bar{n})^{-2 \rho-\mu} d \bar{n} \\
& =\int_{\bar{N} \cap H A N} a_{H}(\bar{n})^{-(2 \rho+\mu)} d \bar{n} \\
& =c_{\mathcal{M}}(\mu+\rho) .
\end{aligned}
$$

That implies,

$$
c_{0}=\frac{\left\langle v_{\mu} \mid \psi_{\mu}\right\rangle}{c_{\mathcal{M}}(\mu+\rho)} .
$$

Lemma 5.1. For all $\gamma \in \Gamma(C)$,

$$
\left\langle\pi_{\mu}\left(\gamma^{-1}\right) \psi_{\mu} \mid \psi_{\mu}\right\rangle=\frac{\left|\left\langle\psi_{\mu} \mid v_{\mu}\right\rangle\right|^{2}}{c_{\mathcal{M}}(\mu+\rho)} \varphi_{-\mu}\left(\gamma^{-1}\right)
$$

Proof. By [10], for all $\gamma \in \Gamma\left(C^{0}\right) \cap G^{c}$ there exist $\bar{n} \in \bar{N}, a_{H}(\gamma) \in A$, and $h \in H$, such that $\gamma=\bar{n} a_{H}(\gamma) h$. Hence for $\gamma \in \Gamma\left(C^{0}\right) \cap G^{c}$,

$$
\left\langle\pi_{\mu}\left(\gamma^{-1}\right) \psi_{\mu} \mid v_{\mu}\right\rangle=a_{H}\left(\gamma^{-1}\right)^{\mu}\left\langle\psi_{\mu} \mid v_{\mu}\right\rangle \text {. }
$$

The equalities (7) and (8) yield

$$
\begin{aligned}
\left\langle\pi_{\mu}\left(\gamma^{-1}\right) \psi_{\mu} \mid \psi_{\mu}\right\rangle & =\frac{\left\langle v_{\mu} \mid \psi_{\mu}\right\rangle}{c_{\mathcal{M}}(\mu+\rho)} \int_{H}\left\langle\pi_{\mu}\left(h \gamma^{-1}\right) \psi_{\mu} \mid v_{\mu}\right\rangle d h \\
& =\frac{\left\langle v_{\mu} \mid \psi_{\mu}\right\rangle}{c_{\mathcal{M}}(\mu+\rho)} \int_{H} a_{H}\left(h \gamma^{-1}\right)^{\mu}\left\langle\psi_{\mu} \mid v_{\mu}\right\rangle d h \\
& =\frac{\left|\left\langle\psi_{\mu} \mid v_{\mu}\right\rangle\right|^{2}}{c_{\mathcal{M}}(\mu+\rho)} \varphi_{-\mu}\left(\gamma^{-1}\right) .
\end{aligned}
$$

Now the assertion follows from the fact that the function $\gamma \mapsto\left\langle\pi_{\mu}\left(\gamma^{-1}\right) \psi_{\mu} \mid \psi_{\mu}\right\rangle$ is holomorphic on $\Gamma\left(C^{0}\right)$ and coincides with $\frac{\left|\left\langle\psi_{\mu} \mid v_{\mu}\right\rangle\right|^{2}}{c_{\mathcal{M}}(\mu+\rho)} \varphi_{-\mu}\left(\gamma^{-1}\right)$ in $\Gamma\left(C^{0}\right) \cap$ $G^{c}$. 
Let $\left(\pi_{\mu}, \mathcal{W}_{\mu}\right)$ be an $H$-spherical unitary highest weight representation of $\widetilde{G}$ such that $\left(\pi_{\mu}, \mathcal{W}_{\mu}\right)$ belongs to the relative discrete series, and

$$
\int_{G / H}\left|\left\langle\pi_{\mu}(g) v_{\mu} \mid \psi_{\mu}\right\rangle\right|^{2} d \dot{g}=\frac{1}{\delta_{\mu}}
$$

where $\delta_{\mu}$ is the relative formal dimension calculated in [20], $\delta_{\mu}=d_{\mu} \cdot c_{\mathcal{M}}(\mu+$ $\rho)$, with $d_{\mu}=\prod_{\beta \in \triangle^{+}} \frac{\langle\mu+\rho, \beta\rangle}{\langle\rho, \beta\rangle}$ the formal dimension of the representation $\pi_{\mu}$.

Theorem 5.1. Let $P_{\nu}$ be the $G$-invariant function such that $P_{\nu}(\exp (2 X)$. ie) $:=p_{\nu}(\exp (X) \cdot i e)$. The weight $\mu \in \mathcal{R}_{\nu, H}^{\prime}$ if and only if the spherical Laplace transform $\widehat{P}_{\nu}(-\mu)$ is finite. Moreover

$$
C_{\mu}(\nu)=\frac{1}{\delta_{\mu}} \widehat{P}_{\nu}(-\mu)
$$

where $\delta_{\mu}$ is the relative formal dimension.

Proof. We assume that the $H$-invariant distribution vector $\psi_{\mu}$ is normalized by $\left\langle\psi_{\mu} \mid v_{\mu}\right\rangle=1$. By the integral formula of Theorem 2.3 and the fact that $p_{\nu}$ is $G$-invariant, we deduce that

$$
\begin{aligned}
C_{\mu}(\nu) & =\int_{\Xi}\left|\left\langle\pi_{\mu}\left(\gamma^{-1}\right) v_{\mu} \mid \psi_{\mu}\right\rangle\right|^{2} p_{\nu}(\xi) d \xi \\
& =\int_{G} \int_{C^{+}}\left|\left\langle\pi_{\mu}\left(\gamma^{-1}\right) v_{\mu} \mid \psi_{\mu}\right\rangle\right|^{2} p_{\nu}(\exp (X) \cdot i e) \prod_{\beta \in \triangle^{+}} \operatorname{sh}(2 \beta(X))^{m_{\beta}} d g d X \\
& =\frac{1}{d_{\mu}} \int_{C^{+}}\left\|\pi_{\mu}(\exp (X)) \psi_{\mu}\right\|^{2} p_{\nu}(\exp (X) \cdot i e) \prod_{\beta \in \triangle^{+}} \operatorname{sh}(2 \beta(X))^{m_{\beta}} d X
\end{aligned}
$$

According to the last lemma, this yields

$$
\begin{aligned}
& \int_{\Xi}\left|\left\langle\pi_{\mu}(\gamma) \psi_{\mu} \mid v_{\mu}\right\rangle\right|^{2} p_{\nu}(\xi) d \xi \\
& =\frac{1}{\delta_{\mu}} \int_{C^{+}} p_{\nu}(\exp (X) \cdot i e) \varphi_{-\mu}(\exp (2 X)) \prod_{\beta \in \Delta^{+}} \operatorname{sh}(2 \beta(X))^{m_{\beta}} d X \\
& =\frac{1}{\delta_{\mu}} \widehat{P}_{\nu}(-\mu) .
\end{aligned}
$$

For any $x \in \mathbb{V}$ we have $x=x^{+}+x^{-}$, where $x^{+}:=\frac{x+\alpha(x)}{2} \in \mathbb{V}^{+}$and $x^{-}:=\frac{x-\alpha(x)}{2} \in \mathbb{V}^{-}$. We denote $x:=\left(x^{+}, x^{-}\right)$. 
Let $\mathcal{J}$ be the bounded set in $\mathbb{V}$ defined by

$$
\begin{aligned}
\mathcal{J}=\left\{x=\left(x^{+}, x^{-}\right) \in \mathbb{V} \mid x^{+} \in \Omega, x^{+}+x^{-} \in e-\bar{\Omega}\right\} \subset & \\
& \left(\mathbb{V}^{+} \cap \Omega\right) \times\left(\left(e+\mathbb{V}^{-}\right) \cap \Omega\right) .
\end{aligned}
$$

Proposition 5.2. The set $\mathcal{J}$ coincides with

$$
\left\{x \in \mathcal{M} \mid x \geq x_{0}\right\}
$$

the future of $x_{0}$.

Proof. Let $\varphi:[0,1] \longrightarrow \mathbb{V}$ be the curve defined by

$$
\varphi(t)=t x+(1-t) e,
$$

where $\varphi(0)=e$ and $\varphi(1)=x$. Since $\Omega$ is convex,

$$
\varphi(t)+\alpha(\varphi(t))=t(x+\alpha(x))+(1-t) 2 e \in \Omega .
$$

Thus $\varphi(t) \in \mathcal{M}$ for all $t \in[0,1]$. Moreover $\dot{\varphi}(t)=x-e \in-\bar{\Omega} \simeq C^{c}$, where

$$
C^{c}=\{(v, 0,-\alpha(v)) \mid v \in \bar{\Omega}\} \subset \mathfrak{g}^{c},
$$

the regular cone in $\mathfrak{g}^{c}$ such that $C^{c} \cap \mathfrak{p}^{c} \neq \emptyset$, where $\mathfrak{p}^{c}=\{(v, L(w),-v) \mid v \in$ $\left.\mathbb{V}^{+}, w \in \mathbb{V}^{-}\right\}$(cf. [3], p. 26). Then, $\varphi$ is a nontrivial causal curve in $\mathcal{M}$ from $x$ to $x_{0}$ and $x$ belongs to the future of $x_{0}$.

Conversely, let $\varphi:[a, b] \longrightarrow \mathcal{M}$ be a causal curve. Assume that there exists $t>a$ such that $\varphi(t) \notin \mathcal{M} \cap \mathbb{V}$ and

$$
\kappa=\inf \{t \in[a, b] \mid \varphi(t) \notin \mathcal{M} \cap \mathbb{V}\} .
$$

Since $\mathcal{M} \cap \mathbb{V}$ is open in $\mathcal{M}$, then $\varphi(t) \in \mathcal{M} \cap \mathbb{V}$ if $t<\kappa$ and $\varphi(t) \notin \mathcal{M} \cap \mathbb{V}$ if not. Hence

$$
\lim _{\substack{t \rightarrow \kappa \\ t<\kappa}}\|\varphi(t)\|=\infty .
$$

Moreover the curve $\varphi:[a, \kappa[\longrightarrow \mathbb{V}$ is causal with respect to the causal structure defined by the cone $-\bar{\Omega}$. Then, $\varphi(t) \in e-\bar{\Omega}$ and for all $t \in[a, \kappa[$, $\varphi(t)$ belongs to the connected component of $x_{0}$ in $\mathcal{M} \cap \mathbb{V}$ given by

$$
\{x \in \mathbb{V} \mid x+\alpha(x) \in \Omega\} .
$$

Hence for all $t \in[a, \kappa[, \varphi(t) \in \mathcal{J}$ such that $\mathcal{J}$ is a bounded set. This leads to contradiction.

Lemma 5.3. Let $\mathbf{s} \in \mathbb{C}^{r^{+}}$and $x=n \exp A(x) \cdot x_{0} \in N A \cdot x_{0}$. To identify $\mathfrak{a}_{\mathbb{C}}$ with $\mathbb{C}^{r^{+}}$we have

$$
e^{\langle\mathbf{s} \mid A(x)\rangle}=\Delta_{\mathbf{s}}\left(\frac{x+\alpha(x)}{2}\right)
$$


Proof. The function $x \mapsto \Delta_{\mathbf{S}}\left(\frac{x+\alpha(x)}{2}\right)$ is $N$-invariant. Then, for all $x=$ $n \exp A(x) \cdot x_{0}$,

$$
\Delta_{\mathbf{S}}\left(\frac{x+\alpha(x)}{2}\right)=\Delta_{\mathbf{s}}\left(\exp A(x) \cdot x_{0}\right) .
$$

Since $A(x) \in \mathfrak{a}_{\mathbb{C}}$, there exists $\left(t_{1}, t_{2}, \ldots, t_{r^{+}}\right) \in \mathbb{C}^{r^{+}}$such that $A(x)=$ $\sum_{j=1}^{r^{+}} t_{j} L\left(e_{j}\right)$, and $\exp A(x) \cdot x_{0}=\sum_{j=1}^{r^{+}} e^{t_{j}} e_{j}$. Here $\left\{e_{j}\right\}_{1 \leq j \leq r^{+}}$is the Jordan frame of $R^{+}$. Thus for all $\mathbf{s}=\left(s_{1}, \ldots, s_{r^{+}}\right)$, we deduce that

$$
\Delta_{\mathbf{s}}\left(\frac{x+\alpha(x)}{2}\right)=\Delta_{\mathbf{s}}\left(\sum_{j=1}^{r^{+}} e^{t_{j}} e_{j}\right)=\prod_{j=1}^{r^{+}} e^{t_{j} s_{j}}=e^{\langle\mathbf{s} \mid A(x)\rangle}
$$

To give an explicit formula of the spherical Laplace transform $\widehat{P}_{\nu}(-\mu)$ and an explicit descripition of the spectrum $\mathcal{R}_{\nu, H}^{\prime}$, we recall some notations.

The Gindikin gamma function of the symmetric cone $\Omega$ is defined by the following integral, generalizing the classical Siegel integral

$$
\begin{aligned}
\Gamma_{\Omega}(\mathbf{s}) & =\int_{\Omega} e^{-\operatorname{tr}(x)} \Delta_{\mathbf{s}-\frac{n}{r}}(x) d x \\
& =\prod_{j=1}^{r} \Gamma\left(s_{j}-\frac{d}{2}(j-1)\right), \quad d:=\frac{2(n-r)}{r(r-1)}
\end{aligned}
$$

for $\mathbf{s}=\left(s_{1}, \ldots, s_{r}\right)$ where $\Re s_{j}>(j-1) \frac{d}{2}$ for $j=1, \ldots, r$.

The Gindikin beta function of the symmetric cone $\Omega$ is defined by the following integral

$$
\begin{aligned}
\mathcal{B}_{\Omega}(\mathbf{s}, \mathbf{m}) & =\int_{\Omega \cap(e-\Omega)} \Delta_{\mathbf{s}-\frac{n}{r}}(x) \Delta_{\mathbf{m}-\frac{n}{r}}(e-x) d x \\
& =\frac{\Gamma_{\Omega}(\mathbf{s}) \Gamma_{\Omega}(\mathbf{m})}{\Gamma_{\Omega}(\mathbf{s}+\mathbf{m})} .
\end{aligned}
$$

Case (I). $\mathbb{V}^{+}$is a simple Jordan algebra.

Let $n^{ \pm}:=\operatorname{dim}\left(\mathbb{V}^{ \pm}\right)$, and $\Gamma_{\Omega^{+}}\left(\operatorname{resp} . \mathcal{B}_{\Omega^{+}}\right)$be the Gindikin gamma (resp. beta) function of the symmetric cone $\Omega^{+}:=\Omega \cap \mathbb{V}^{+}$of $\mathbb{V}^{+}$.

Lemma 5.4. For $\lambda \in \mathbb{C}$ such that $\Re(\lambda)>\frac{n}{r}-1$,

$$
\int_{(-e+\Omega) \cap \mathbb{V}^{-}} \Delta(e+v)^{\frac{r}{r^{+}} \lambda-\frac{n}{r^{+}}} d v=\frac{\Gamma_{\Omega}(\lambda)}{\Gamma_{\Omega^{+}}\left(\frac{r}{r^{+}} \lambda\right)} .
$$


Proof. With respect to the decomposition of the Jordan algebra $\mathbb{V}$ as $\mathbb{V}=$ $\mathbb{V}^{+} \oplus \mathbb{V}^{-}$, the Gindikin gamma function of the symmetric cone $\Omega$ can be written as

$$
\begin{aligned}
\Gamma_{\Omega}(\lambda) & =\int_{\Omega} e^{-\operatorname{tr}(x)} \Delta(x)^{\lambda-\frac{n}{r}} d x \\
& =\int_{\Omega^{+}} e^{-\operatorname{tr}\left(x^{+}\right)}\left[\int_{\left\{x^{-} \mid x^{+}+x^{-} \in \Omega\right\}} \Delta\left(x^{+}+x^{-}\right)^{\lambda-\frac{n}{r}} d x^{-}\right] d x^{+} .
\end{aligned}
$$

Moreover $x^{+}+x^{-}=P\left(\left(x^{+}\right)^{\frac{1}{2}}\right)(e+v)$, where $P$ is the quadratic representation and $v=P\left(\left(x^{+}\right)^{-\frac{1}{2}}\right) x^{-}$. Hence

$$
\begin{aligned}
\Gamma_{\Omega}(\lambda) & =\int_{\Omega^{+}} e^{-t r\left(x^{+}\right)} \Delta\left(x^{+}\right)^{\frac{r}{r^{+}} \lambda-\frac{n}{r^{+}}+\frac{n^{-}}{r^{+}}} d x^{+} \int_{(-e+\Omega) \cap \mathbb{V}^{-}} \Delta(e+v)^{\frac{r}{r^{+}} \lambda-\frac{n}{r^{+}}} d v \\
& =\Gamma_{\Omega^{+}}\left(\frac{r}{r^{+}} \lambda\right) \int_{(-e+\Omega) \cap \mathbb{V}^{-}} \Delta(e+v)^{\frac{r}{r^{+}} \lambda-\frac{n}{r^{+}}} d v .
\end{aligned}
$$

This proves that

$$
\int_{(-e+\Omega) \cap \mathbb{V}^{-}} \Delta(e+v)^{\frac{r}{r^{+}} \lambda-\frac{n}{r^{+}}} d v=\frac{\Gamma_{\Omega}(\lambda)}{\Gamma_{\Omega^{+}}\left(\frac{r}{r^{+}} \lambda\right)} .
$$

Proposition 5.5. For $\mu=\left(m_{1}+\left(r / 2 r^{+}\right) \nu, \ldots, m_{r^{+}}+\left(r / 2 r^{+}\right) \nu\right)$, the spherical Laplace transform $\widehat{P}_{\nu}(-\mu)$ is finite if and only if

$$
m_{1} \geq m_{2} \geq \cdots \geq m_{r^{+}} \geq 0 .
$$

Moreover

$$
\widehat{P}_{\nu}(-\mu)=c_{0} \Gamma_{\Omega}\left(\nu-\frac{n}{r}\right) \frac{\Gamma_{\Omega^{+}}\left(\mu-\nu \frac{r}{2 r^{+}}+\frac{n^{+}}{r^{+}}\right)}{\Gamma_{\Omega^{+}}\left(\mu+\nu \frac{r}{2 r^{+}}-\frac{n^{-}}{r^{+}}\right)},
$$

where $c_{0}$ is a positive constant.

Proof. By Proposition 3.1 and for all $x=n \exp A(x) \cdot x_{0}$, the function $p_{\nu}$ is given by

$$
p_{\nu}(x)=\operatorname{det}(P(x+\alpha(x)))^{-\frac{r}{2 n} \nu+1} \Delta\left(e-x^{2}\right)^{\nu-\frac{2 n}{r}} .
$$

Since $A(x) \in \mathfrak{a}_{\mathbb{C}}$, there exists $\left(t_{1}, \ldots, t_{r^{+}}\right) \in \mathbb{C}^{r^{+}}$such that $A(x)=$ $\sum_{j=1}^{r^{+}} t_{j} L\left(e_{j}\right)$, and

$$
\exp \left(\frac{A(x)}{2}\right) \cdot x_{0}=\sum_{j=1}^{r^{+}} e^{\frac{t_{j}}{2}} e_{j}
$$


Let $x=\sum_{j=1}^{r^{+}} e^{t_{j}} e_{j}$ and $u=\sum_{j=1}^{r^{+}} e^{\frac{t_{j}}{2}} e_{j}$. Using the fact that $p_{\nu}$ is $G$ invariant, we deduce that

$$
p_{\nu}(u)=\Delta\left(x^{+}\right)^{-\nu \frac{r}{2 r^{+}}+\frac{n}{r^{+}}} \Delta(e-x)^{\nu-\frac{2 n}{r}}=P_{\nu}(x) .
$$

For $x \in \mathbb{V}$, let $d \delta(x)$ be the $G$-invariant measure on $\mathcal{M}$, where its restriction to $\mathcal{M} \cap \mathbb{V}$ is equal to $d \delta(x)=\Delta\left(x^{+}\right)^{-\frac{n}{r^{+}}} d x^{+} d x^{-}$. Using Lemma 5.3, the spherical Laplace transformation of $P_{\nu}$ can be written as

$$
\begin{aligned}
& \widehat{P}_{\nu}(-\mu)=\int_{x \geq x_{0}} e^{\langle\mu, A(x)\rangle} P_{\nu}(x) d \delta(x) \\
& =\int_{\Omega^{+} \cap\left(e-\Omega^{+}\right)} \int_{\left\{x^{-} \mid e-x^{+}-x^{-} \in \Omega\right\}} \Delta_{\mu}\left(x^{+}\right) \Delta\left(x^{+}\right)^{-\nu \frac{r}{2 r^{+}}+\frac{n}{r^{+}}} \\
& \cdot \Delta(e-x)^{\nu-\frac{2 n}{r}} \Delta\left(x^{+}\right)^{-\frac{n}{r^{+}}} d x^{+} d x^{-} \\
& =\int_{\Omega^{+} \cap\left(e-\Omega^{+}\right)} \Delta_{\mu-\nu \frac{r}{2 r^{+}}}\left(x^{+}\right) \\
& \cdot\left[\int_{\left\{x^{-} \mid e-x^{+}-x^{-} \in \Omega\right\}} \Delta\left(e-x^{+}-x^{-}\right)^{\nu-\frac{2 n}{r}} d x^{-}\right] d x^{+} .
\end{aligned}
$$

Using the last lemma, and the fact that $e-x^{+}-x^{-}=P\left(\left(e-x^{+}\right)^{\frac{1}{2}}\right)(e-v)$ where $v=P\left(\left(e-x^{+}\right)^{-\frac{1}{2}}\right) x^{-}$, we obtain

$$
\begin{aligned}
\widehat{P}_{\nu}(-\mu)= & \int_{\Omega^{+} \cap\left(e-\Omega^{+}\right)} \Delta_{\mu-\nu \frac{r}{2 r^{+}}}\left(x^{+}\right) \Delta\left(e-x^{+}\right)^{\nu \frac{r}{r^{+}}-\frac{2 n}{r^{+}}+\frac{n^{-}}{r^{+}}} d x^{+} \\
& \cdot \int_{(e-\Omega) \cap \mathbb{V}^{-}} \Delta(e-v)^{\nu \frac{r}{r^{+}}-\frac{2 n}{r^{+}}} d v \\
= & \frac{\Gamma_{\Omega}\left(\nu-\frac{n}{r}\right)}{\Gamma_{\Omega^{+}}\left(\nu \frac{r}{r^{+}}-\frac{n}{r^{+}}\right)} \mathcal{B}_{\Omega^{+}}\left(\mu-\nu \frac{r}{2 r^{+}}+\frac{n^{+}}{r^{+}}, \nu \frac{r}{r^{+}}-\frac{n}{r^{+}}\right),
\end{aligned}
$$

such that $\mu_{j}-\nu \frac{r}{2 r^{+}}+\frac{n^{+}}{r^{+}}>(j-1) \frac{n^{+}-r^{+}}{r^{+}\left(r^{+}-1\right)}$ for $1 \leq j \leq r^{+}$. By the fact that $\mu_{j}=m_{j}+\nu \frac{r}{2 r^{+}}$, where $m_{j} \in \mathbb{Z}$ and $\mu_{j}-\mu_{j+1} \in \mathbb{N}$, this condition will be written as

$$
m_{1} \geq m_{2} \geq \cdots \geq m_{r^{+}} \geq 0
$$


It follows from Proposition 5.5 that the spectrum of the Bergman space $\mathcal{H}_{\nu}^{2}(\widetilde{\Xi})$ is given by

$$
\begin{array}{r}
\mathcal{R}_{\nu, H}^{\prime}=\left\{\mu=\left(m_{1}+\left(r / 2 r^{+}\right) \nu, m_{2}+\left(r / 2 r^{+}\right) \nu, \ldots, m_{r^{+}}+\left(r / 2 r^{+}\right) \nu\right) \mid\right. \\
\left.m_{1} \geq m_{2} \geq \cdots \geq m_{r^{+}} \geq 0\right\} .
\end{array}
$$

Recall that the constant $c_{\nu}=\frac{1}{\pi^{n}} \frac{\Gamma_{\Omega}(\nu)}{\Gamma_{\Omega}\left(\nu-\frac{n}{r}\right)}$ (see (4)), the reproducing kernel $K_{\nu}\left(\widetilde{\xi}_{1}, \widetilde{\xi}_{2}\right)$ can be written as

$$
\begin{aligned}
& \top_{\alpha}\left(\widetilde{\xi}_{1}\right)^{\frac{r}{4 n} \nu} \operatorname{det}\left(B\left(\xi_{1}, \bar{\xi}_{2}\right)\right)^{-\frac{r}{2 n} \nu} \overline{T_{\alpha}\left(\widetilde{\xi}_{2}\right)^{\frac{r}{4 n}} \nu} \\
& =\sum \frac{c_{0} d_{\mu}}{\Gamma_{\Omega}(\nu)} \frac{\Gamma_{\Omega^{+}}\left(\mathbf{m}+\nu \frac{r}{r^{+}}-\frac{n^{-}}{r^{+}}\right)}{\Gamma_{\Omega^{+}}\left(\mathbf{m}+\frac{n^{+}}{r^{+}}\right)} \varphi_{-\mu}\left(\left(\widetilde{\gamma}_{2}^{\sharp} \widetilde{\gamma}_{1}\right)^{-1}\right),
\end{aligned}
$$

for all $\widetilde{\xi}_{1}=\widetilde{\gamma}_{1} \cdot H$ and all $\widetilde{\xi}_{2}=\widetilde{\gamma}_{2} \cdot H$ in $\widetilde{\Xi}$. The summation index runs over the integers of type $\mathbf{m}:=\left(m_{1}, m_{2}, \ldots, m_{r^{+}}\right)$such that $m_{1} \geq \cdots \geq m_{r^{+}} \geq 0$. We use $\mathbf{m} \geq 0$ to denote the summation index. The function

$$
\nu \mapsto \top_{\alpha}\left(\widetilde{\xi}_{1}\right)^{\frac{r}{4 n}} \nu \operatorname{det}\left(B\left(\xi_{1}, \bar{\xi}_{2}\right)\right)^{-\frac{r}{2 n} \nu} \bar{\top}_{\alpha}\left(\widetilde{\xi}_{2}\right)^{\frac{r}{4 n}} \nu,
$$

is holomorphic on $\mathbb{C}$ and coincides with

$$
\sum_{\mathbf{m} \geq 0} \frac{c_{0} d_{\mu}}{\Gamma_{\Omega}(\nu)} \frac{\Gamma_{\Omega^{+}}\left(\mathbf{m}+\nu \frac{r}{r^{+}}-\frac{n^{-}}{r^{+}}\right)}{\Gamma_{\Omega^{+}}\left(\mathbf{m}+\frac{n^{+}}{r^{+}}\right)} \varphi_{-\mu}\left(\left(\widetilde{\gamma}_{2}^{\sharp} \widetilde{\gamma}_{1}\right)^{-1}\right),
$$

for $\nu \in] \frac{2 n}{r}-1,+\infty[$, therefore these two functions coincide everywhere. The following theorem holds.

Theorem 5.2. Assume $\alpha$ is given such that $\mathbb{V}^{+}$is a simple algebra. Then for all $\nu \in \mathbb{C}$ such that $\Re(\nu)>\frac{n}{r}-1$,

$$
\begin{aligned}
& \top_{\alpha}\left(\widetilde{\xi}_{1}\right)^{\frac{r}{4 n} \nu} \operatorname{det}\left(B\left(\xi_{1}, \bar{\xi}_{2}\right)\right)^{-\frac{r}{2 n} \nu} \overline{\top_{\alpha}\left(\widetilde{\xi}_{2}\right)} \frac{r}{4 n} \nu \\
& =\sum_{\mathbf{m} \geq 0} \frac{c_{0} d_{\mu}}{\Gamma_{\Omega}(\nu)} \frac{\Gamma_{\Omega^{+}}\left(\mathbf{m}+\nu \frac{r}{r^{+}}-\frac{n^{-}}{r^{+}}\right)}{\Gamma_{\Omega^{+}}\left(\mathbf{m}+\frac{n^{+}}{r^{+}}\right)} \varphi_{-\mu}\left(\left(\widetilde{\gamma}_{2}^{\sharp} \widetilde{\gamma}_{1}\right)^{-1}\right) .
\end{aligned}
$$

The series converges uniformly on compact subsets of $\widetilde{\Xi} \times \widetilde{\Xi}$. 
An application (Makarevič symmetric spaces of Cayley type). Let $\mathbb{V}$ be a simple Euclidean Jordan algebra of dimension $n$ and rank $r$. The bounded symmetric domain associated with $\mathbb{V}_{\mathbb{C}} \times \mathbb{V}_{\mathbb{C}}$ is the bidisc $\mathcal{D} \times \mathcal{D}$ where $\mathcal{D}$ is the unit disc of $\mathbb{V}_{\mathbb{C}}$. Let $\alpha$ be the involution on $\mathcal{D} \times \mathcal{D}$ defined by $\alpha(z, w)=$ $(-w,-z)$. In this case, the domain $\Xi$ is realized as $\mathcal{D} \times \mathcal{D} \backslash \Sigma$ where

$$
\Sigma=\{(z, w) \in \mathcal{D} \times \mathcal{D} \mid \Delta(z-w)=0\},
$$

and $\Delta$ is the determinant polynomial associated with $\mathbb{V}$ (see (1)).

Let

$$
\left\{z, w ; z^{\prime}, w^{\prime}\right\}:=\frac{\Delta\left(z^{\prime}-z\right) \Delta\left(w-w^{\prime}\right)}{\Delta(z-w) \Delta\left(z^{\prime}-w^{\prime}\right)}
$$

be the cross-ratio of four points $z, w, z^{\prime}$, and $w^{\prime}$ of $\mathbb{V}_{\mathbb{C}}$. This definition generalizes the classical cross-ratio-matrix and satisfies the $G$-invariance property $\left\{g \cdot z, g \cdot w ; g \cdot z^{\prime}, g \cdot w^{\prime}\right\}=\left\{z, w ; z^{\prime}, w^{\prime}\right\}$. The reproducing kernel of the Bergman space $\mathcal{H}_{\nu}^{2}(\widetilde{\Xi})$ is equal to

$$
K_{\nu}\left(\widetilde{z}_{1}, \widetilde{w}_{1} ; \widetilde{z}_{2}, \widetilde{w}_{2}\right)=c_{\nu}^{2}\left\{\widetilde{z}_{1}, \widetilde{w}_{1} ; \overline{\widetilde{z}}_{2}^{-1},{\widetilde{w_{2}}}^{-1}\right\}^{\nu},
$$

for $\left(\widetilde{z}_{1}, \widetilde{w}_{1}\right)$ and $\left(\widetilde{z}_{2}, \widetilde{w}_{2}\right)$ in $\widetilde{\Xi}$.

Using Theorem 5.2, we deduce a formula of a complex power of a crossratio of four points,

$$
\left\{\widetilde{z}_{1}, \widetilde{w}_{1} ;{\widetilde{z_{2}}}^{-1},{\widetilde{w_{2}}}^{-1}\right\}^{\nu}=\sum_{\mathbf{m} \geq 0} \frac{c_{0} d_{\mu}}{\Gamma_{\Omega}(\nu)^{2}} \frac{\Gamma_{\Omega}\left(\mathbf{m}+2 \nu-\frac{n}{r}\right)}{\Gamma_{\Omega}\left(\mathbf{m}+\frac{n}{r}\right)} \varphi_{-\mu}\left(\left(\widetilde{\gamma}_{2}^{\sharp} \widetilde{\gamma}_{1}\right)^{-1}\right),
$$

for all $\left(\widetilde{z}_{1}, \widetilde{w}_{1}\right)=\widetilde{\gamma}_{1} \cdot H$ and all $\left(\widetilde{z}_{2}, \widetilde{w}_{2}\right)=\widetilde{\gamma}_{2} \cdot H$. We remark that this formula is a generalization of that given in Theorem 3.1 of [7].

Let $\mathcal{W}$ be the Wallach set

$$
\left.\mathcal{W}=\left\{0, \frac{n-r}{r(r-1)}, \ldots, \frac{n}{r}-1\right\} \cup\right] \frac{n}{r}-1,+\infty[,
$$

(cf. [12] p. 268), and let $\mathcal{K}_{\nu}\left(\widetilde{z}_{1}, \widetilde{w}_{1} ; \widetilde{z}_{2}, \widetilde{w}_{2}\right):=\left\{\widetilde{z}_{1}, \widetilde{w}_{1} ;{\widetilde{\widetilde{z}_{2}}}^{-1},{\widetilde{\widetilde{w}_{2}}}^{-1}\right\}^{\nu}$. The kernel $\mathcal{K}_{\nu}$ is of positive type on $\widetilde{\Xi} \times \widetilde{\Xi}$ if and only if $\nu$ belongs to $\mathcal{W}$. Then $\mathcal{K}_{\nu}$ is a reproducing kernel of some Hilbert space $\mathcal{H}_{\nu}(\widetilde{\Xi})$. In particular if $\nu>\frac{2 n}{r}-1, \mathcal{H}_{\nu}(\widetilde{\Xi})=\mathcal{H}_{\nu}^{2}(\widetilde{\Xi})$.

We note that $\frac{n}{r} \in \frac{1}{2} \mathbb{N}$. In $[\mathbf{1 2}]$, J. Faraut and A. Korányi showed that the analytic continuation Bergman space $\mathcal{H}_{\frac{n}{r}}(\mathcal{D} \times \mathcal{D})\left(\simeq \mathcal{H}_{\frac{n}{r}}(\widetilde{\Xi})\right.$ by Theorem 3.1) coincides with the Hardy space $H^{2}(\mathcal{D} \times \mathcal{D})$. It is a Hilbert space of holomorphic functions $f$ on $\mathcal{D} \times \mathcal{D}$ such that

$$
\sup _{0<r_{1}, r_{2}<1} \int_{\mathcal{S} \times \mathcal{S}}\left|f\left(r_{1} u_{1}, r_{2} u_{2}\right)\right|^{2} d \sigma\left(u_{1}\right) d \sigma\left(u_{2}\right)<\infty,
$$


where $\mathcal{S}$ is the Shilov boundary of $\mathcal{D}$.

Using the same notations as in Section 3, the covering $\widetilde{G}$ of order 2 of $G$ acts on $H^{2}(\mathcal{D} \times \mathcal{D})$ by the representation $\widetilde{\pi}_{\frac{n}{r}}:=\pi_{\frac{n}{r}} \otimes \pi_{\frac{n}{r}}:$ for $f \in H^{2}(\mathcal{D} \times \mathcal{D})$ and $\widetilde{g}^{-1}=(g, \varphi) \in \widetilde{G}$,

$$
\left(\widetilde{\pi}_{\frac{n}{r}}(\widetilde{g}) f\right)(z, w)=\left(\frac{\Delta(g \cdot z-g \cdot w)}{\Delta(z-w)}\right)^{\frac{n}{r}} f(g \cdot z, g \cdot w) .
$$

Using [4] Theorem 3.2.1, we can deduce that the representation $\widetilde{\pi}_{\frac{n}{r}}$ decomposes into a discrete sum of irreducible unitary representations $\pi_{\mu}$ of $\widetilde{G}$ with highest weights

$$
\mu=\left(m_{1}+\frac{n}{r}, m_{2}+\frac{n}{r}, \ldots, m_{r}+\frac{n}{r}\right)
$$

such that $m_{1} \geq m_{2} \geq \cdots \geq m_{r} \geq 0$. In particular, the decomposition of the Hardy space $H^{2}(\mathcal{D} \times \mathcal{D})$ holds. Hence, we find the decomposition of $H^{2}(\mathcal{D} \times \mathcal{D})$ shown in $[7]$ when $\frac{n}{r}$ belongs to $\mathbb{N}$.

\section{Case (II): $\mathbb{V}^{+}$is a direct sum of two simple algebras.}

Let $\mathbb{V}^{+}$be a direct sum of two simple algebras $\mathbb{V}_{1}^{+}$of rank $p$ and $\mathbb{V}_{2}^{+}$of rank $q$ such that $p+q=r^{+}(=r)$. Let $n_{1}=p+\frac{1}{2} d p(p-1)$ denote the dimension of $\mathbb{V}_{1}^{+}$, and $n_{2}=q+\frac{1}{2} d q(q-1)$ the dimension of $\mathbb{V}_{2}^{+}$. Then the involution $\alpha$ is given by $\alpha=P(w)$, where $w=c_{1}+c_{2}+\cdots+c_{p}-c_{p+1}-c_{p+2}-\cdots-c_{r}$. The cone $\Omega^{+}$is a direct sum of $\Omega_{1}^{+}$and $\Omega_{2}^{+}$, where $\Omega_{1}^{+}:=\Omega^{+} \cap \mathbb{V}_{1}^{+}$and $\Omega_{2}^{+}:=\Omega^{+} \cap \mathbb{V}_{2}^{+}$.

For $\mathbf{s} \in \mathbb{C}^{p}\left(\right.$ resp. $\left.\mathbb{C}^{q}\right)$, we write $\Delta_{\mathbf{s}}^{(1)}\left(\right.$ resp. $\left.\Delta_{\mathbf{s}}^{(2)}\right)$ for the generalized power function associated with $\Omega_{1}^{+}\left(\operatorname{resp} . \Omega_{2}^{+}\right)$.

Using the same techniques as in Lemma 5.4, we show that for all $\lambda \in \mathbb{C}$ such that $\Re(\lambda)>\frac{n}{r}-1$,

$$
\int_{(-e+\Omega) \cap \mathbb{V}^{-}} \Delta(e+v)^{\lambda-\frac{n}{r}} d v=\frac{\Gamma_{\Omega}(\lambda)}{\Gamma_{\Omega_{1}^{+}}\left(\lambda-\frac{n^{+}}{r}+\frac{n_{1}}{p}\right) \Gamma_{\Omega_{2}^{+}}\left(\lambda-\frac{n^{+}}{r}+\frac{n_{2}}{q}\right)} .
$$

Here $\Gamma_{\Omega_{1}^{+}}$(resp. $\Gamma_{\Omega_{2}^{+}}$) denotes the Gindikin gamma function associated with the symmetric cone $\Omega_{1}^{+}$(resp. $\Omega_{2}^{+}$).

Proposition 5.6. For all $\mu=\left(m_{1}+(\nu / 2), \ldots, m_{p}+(\nu / 2) ; m_{p+1}+(\nu / 2)\right.$, $\left.\ldots, m_{r}+(\nu / 2)\right):=\left(\underline{\mu}_{1} ; \underline{\mu}_{2}\right)$, the spherical Laplace transform $\widehat{P}_{\nu}(-\mu)$ is finite if and only if

$$
m_{1} \geq \cdots \geq m_{p} \geq 0, \quad m_{p+1} \geq \cdots \geq m_{r} \geq 0 .
$$


Moreover

$$
\begin{aligned}
\widehat{P}_{\nu}(-\mu)= & c_{0} \Gamma_{\Omega}\left(\nu-\frac{n}{r}\right) \\
& \cdot \frac{\Gamma_{\Omega_{1}^{+}}\left(\underline{\mu}_{1}-\frac{\nu}{2}+\frac{n_{1}}{p}\right) \Gamma_{\Omega_{2}^{+}}\left(\underline{\mu}_{2}-\frac{\nu}{2}+\frac{n_{2}}{q}\right)}{\Gamma_{\Omega_{1}^{+}}\left(\underline{\mu}_{1}+\frac{\nu}{2}-\frac{n^{+}}{r}-\frac{n}{r}+\frac{2 n_{1}}{p}\right) \Gamma_{\Omega_{2}^{+}}\left(\underline{\mu}_{2}+\frac{\nu}{2}-\frac{n^{+}}{r}-\frac{n}{r}+\frac{2 n_{2}}{q}\right)},
\end{aligned}
$$

where $c_{0}$ is a positive constant.

Proof. As proved in Proposition 5.5, we have

$$
\begin{aligned}
\widehat{P}_{\nu}(-\mu)= & \int_{\Omega_{1}^{+} \cap\left(e-\Omega_{1}^{+}\right)} \Delta_{\underline{\mu}_{1}-\frac{\nu}{2}}^{(1)}\left(x_{1}^{+}\right) \Delta^{(1)}\left(e-x_{1}^{+}\right)^{\nu+\frac{n^{-}}{r}-\frac{2 n}{r}} d x_{1}^{+} \\
& \int_{\Omega_{2}^{+} \cap\left(e-\Omega_{2}^{+}\right)} \Delta_{\underline{\mu}_{2}-\frac{\nu}{2}}^{(2)}\left(x_{2}^{+}\right) \Delta^{(2)}\left(e-x_{2}^{+}\right)^{\nu+\frac{n^{-}}{r}-\frac{2 n}{r}} d x_{2}^{+} \\
& \int_{(e-\Omega) \cap \mathbb{V}-} \Delta(e-v)^{\nu-\frac{2 n}{r}} d v \\
= & \mathcal{B}_{\Omega_{1}^{+}}\left(\underline{\mu}_{1}-\frac{\nu}{2}+\frac{n_{1}}{p}, \nu-\frac{n^{+}}{r}-\frac{n}{r}+\frac{n_{1}}{p}\right) \\
& \mathcal{B}_{\Omega_{2}^{+}}\left(\underline{\mu}_{2}-\frac{\nu}{2}+\frac{n_{2}}{q}, \nu-\frac{n^{+}}{r}-\frac{n}{r}+\frac{n_{2}}{q}\right) \\
& \int_{(e-\Omega) \cap \mathbb{V}^{-}} \Delta(e-v)^{\nu-\frac{2 n}{r}} d v .
\end{aligned}
$$

Then the assertion follows from formula (9).

Let $\mathbf{m}:=\left(\mathbf{m}_{1}, \mathbf{m}_{2}\right)=\left(m_{1}, \ldots, m_{p} ; m_{p+1}, \ldots, m_{r}\right)$. We use $\mathbf{m} \geq 0$ to denote $m_{1} \geq \cdots \geq m_{p} \geq 0 ; \quad m_{p+1} \geq \cdots \geq m_{r} \geq 0$. We apply the similar arguments as in Theorem 5.2, the following theorem holds.

Theorem 5.3. Assume $\alpha$ is given such that $\mathbb{V}^{+}$is a direct sum of two simple algebras. Then for all $\nu \in \mathbb{C}$ such that $\Re(\nu)>\frac{n}{r}-1$,

$$
\begin{aligned}
& \top_{\alpha}\left(\widetilde{\xi}_{1}\right)^{\frac{r}{4 n} \nu} \operatorname{det}\left(B\left(\xi_{1}, \bar{\xi}_{2}\right)\right)^{-\frac{r}{2 n} \nu} \overline{T_{\alpha}\left(\widetilde{\xi}_{2}\right)^{\frac{r}{4 n}} \nu} \\
& =\sum_{\mathbf{m} \geq 0} \frac{c_{0} d_{\mu}}{\Gamma_{\Omega}(\nu)} \\
& \quad \cdot \frac{\Gamma_{\Omega_{1}^{+}}\left(\mathbf{m}_{1}+\nu-\frac{n^{+}}{r}-\frac{n}{r}+\frac{2 n_{1}}{p}\right) \Gamma_{\Omega_{2}^{+}}\left(\mathbf{m}_{2}+\nu-\frac{n^{+}}{r}-\frac{n}{r}+\frac{2 n_{2}}{q}\right)}{\Gamma_{\Omega_{1}^{+}}\left(\mathbf{m}_{1}+\frac{n_{1}}{p}\right) \Gamma_{\Omega_{2}^{+}}\left(\mathbf{m}_{2}+\frac{n_{2}}{q}\right)} \varphi_{-\mathbf{m}-\frac{\nu}{2}} \\
& \quad\left(\left(\widetilde{\gamma}_{2}^{\#} \widetilde{\gamma}_{1}\right)^{-1}\right) .
\end{aligned}
$$

The series converges uniformly on compact subsets of $\widetilde{\Xi} \times \widetilde{\Xi}$. 
Remark. If $p=0$ or $q=0$, then $\alpha=\mathrm{id}_{\mathbb{V}}$ (the compact case). The last series can be written as

$$
\Delta(e-z)^{-\nu}=c_{0} \sum_{\mathbf{m} \geq 0} d_{\mathbf{m}} \frac{(\nu)_{\mathbf{m}}}{\left(\frac{n}{r}\right)_{\mathbf{m}}} \varphi_{\mathbf{m}}(z),
$$

with $\mathbf{m}=\left(m_{1}, m_{2}, \ldots, m_{r}\right)$. Here $(\mathbf{s})_{\mathbf{m}}$ denote $(\mathbf{s})_{\mathbf{m}}=\frac{\Gamma_{\Omega}(\mathbf{s}+\mathbf{m})}{\Gamma_{\Omega}(\mathbf{s})}$, for all $\mathbf{s} \in \mathbb{C}^{r}$ and $\mathbf{m} \in \mathbb{N}^{r}$. Hence, we find the generalized binomial formula shown in $[\mathbf{1 2}]$. See also [2].

We present here the table of Makarevič symmetric space $G / H$, and its dual symmetric space $G^{c} / H$.

\begin{tabular}{||l||c|c||}
\hline \multicolumn{1}{||c||}{$\mathbb{V}$} & $G / H$ & $G^{c} / H$ \\
\hline \hline 1$)$ Herm $(n, \mathbb{C})$ & $U(p, q)(\mathrm{p}+\mathrm{q}=\mathrm{n})$ & $G L(n, \mathbb{C}) / U(p, q)$ \\
& $S O^{*}(2 n) / S O(n, \mathbb{C})$ & $S O(n, n) / O(n, \mathbb{C})$ \\
& $S p(4 n, \mathbb{R}) / S p(2 n, \mathbb{C})$ & $S p(n, n) / S p(2 n, \mathbb{C})$ \\
\hline 2) Sym $(n, \mathbb{R})$ & $U(p, q) / O(p, q)$ & $G L(n, \mathbb{R}) / O(p, q)$ \\
& $S p(2 n, \mathbb{R})$ & $S p(2 n, \mathbb{C}) / S p(2 n, \mathbb{R})$ \\
\hline 3) Herm $(n, \mathbb{H})$ & $U(2 p, 2 q) / S p(p, q)$ & $U^{*}(2 n) / S p(p, q)$ \\
& $S O^{*}(2 n)$ & $S O(2 n, \mathbb{C}) / O^{*}(2 n)$ \\
\hline 4) $\mathbb{R} \times \mathbb{R}^{n-1}$ & $S O(p) \times S O(2, q) /$ & $S O(1, p-1) \times S O(1, q+1) /$ \\
& $S O(p-1) \times S O(1, q)$ & $S O(p-1) \times S O(1, q)$ \\
\hline 5$)$ Herm $(3, \mathbb{O})$ & $E_{6} \times U(1) / F_{4}$ & $E_{6} \times \mathbb{R}^{+} / F_{4}$ \\
& $E_{6(-14)} \times U(1) / F_{4(-20)}$ & $E_{6(-14)} \times \mathbb{R}^{+} / F_{4(-20)}$ \\
& $S U(6,2) / S p(3,1)$ & $S U^{*}(8) / S p(3,1)$ \\
\hline $\mathbb{V} \times \mathbb{V}$, where $\mathbb{V}$ is & \multicolumn{2}{|c|}{ Cayley type } \\
either of the type & $S U(n, n) / S L(n, \mathbb{C}) \times \mathbb{R}_{+}^{*}$ & $S U(n, n) / S L(n, \mathbb{C}) \times \mathbb{R}_{+}^{*}$ \\
$1), 2), 3), 4)$, or 5$).$ & $S p(2 n, \mathbb{R}) / G L(n, \mathbb{R})$ & $S p(2 n, \mathbb{R}) / G L(n, \mathbb{R})$ \\
& $S O^{*}(4 n) / S L(n, \mathbb{H}) \times \mathbb{R}_{+}^{*}$ & $S O^{*}(4 n) / S L(n, \mathbb{H}) \times \mathbb{R}_{+}^{*}$ \\
& $S O(2, n) / S O(1, n-1) \times \mathbb{R}_{+}^{*} S O(2, n) / S O(1, n-1) \times \mathbb{R}_{+}^{*}$ & $E_{7(-25)} / E_{6(-26)} \times \mathbb{R}_{+}^{*}$ \\
\hline \hline
\end{tabular}

Acknowledlement. I am very grateful to my supervisor Jacques Faraut who helped and encouraged me with his advice and many fruitful discussions. 


\section{References}

[1] S. Ben Saïd, Espaces de Bergman pondérés et série discrète holomorphe de $\widetilde{U(p, q)}$, J. Funct. Anal., 173 (2000), 154-181, MR 2001g:43011, Zbl 0957.43008.

[2] _ Espaces de Bergman Pondérés sur un Domaine Symétrique Borné, Thèse, Universié Paris VI (2000).

[3] W. Bertram, Algebraic structures of Makarevič spaces I, Transformation Groups, 3 (1998), 3-32, MR 99c:32047, Zbl 0894.22004.

[4] W. Bertram and J. Hilgert, Hardy spaces and analytic continuation of Bergman spaces, Bull. Soc. Math. France, 126 (1998), 435-482, MR 2000a:32012, Zbl 0920.22006.

[5] J.L. Brylinski and P. Delorme, Vecteur distributions H-invariants pour les séries principales généralisées d'espaces symétriques réductifs et prolongement méromorphe d'intégrales d'Eisenstein, Invent. Math., 109 (1992), 619-664, MR 93m:22016, Zbl 0785.22014.

[6] B. Chabat, Introduction à l'Analyse Complexe, Editions MIR, 1990, MR 91k:30002, Zbl 0732.32001.

[7] M. Chadli, Noyau de Cauchy-Szegö d'un espace symétrique de type Cayley, Ann. Inst. Fourier, 48 (1998), 97-132, MR 99b:22022, Zbl 0920.43008.

[8] G. van Dijk and M. Pevzner, Berezin kernels on tube domains, J. Funct. Anal., 181 (2001), 189-208, MR 2002c:32032, Zbl 0970.43003.

[9] J. Faraut, Fonctions sphériques sur un espace symétrique de type Cayley, Contemp. Math., 191 (1995), 41-55, MR 97c:43012, Zbl 0847.53039.

[10] J. Faraut, J. Hilgert and G. Ólafsson, Spherical functions on ordered symmetric spaces, Ann. Inst. Fourier, 44 (1994), 927-966, MR 96a:43012, Zbl 0810.43003.

[11] J. Faraut and A. Korányi, Function spaces and reproducing kernels on bounded symmetric domains, J. Funct. Anal., 88 (1990), 64-89, MR 90m:32049, Zbl 0718.32026.

[12] _ Analysis on Symmetric Cones, Oxford University Press, 1994, MR 98g:17031, Zbl 0841.43002 .

[13] J. Hilgert and B. Krötz, Weighted Bergman spaces associated with causal symmetric spaces, Manuscripta Math., 99 (1999), 151-180, MR 2000g:22019, Zbl 0961.32008.

[14] J. Hilgert, G. Ólafsson and B. Ørsted, Hardy spaces on affine symmetric spaces, J. Reine Angew. Math., 415 (1991), 189-218, MR 92h:22030, Zbl 0716.43006.

[15] T. Kobayashi, Multiplicity free branching laws for unitary highest weight modules, in 'Proceedings of Symposium on representation theory held at Saga', Kyushu (1997), 9-17.

[16] Discrete decomposability of the restriction of $A_{\mathfrak{q}}(\lambda)$ with respect to reductive subgroups, Invent. Math., 131 (1997), 229-256, MR 99k:22021, Zbl 0907.22016.

[17] _ Discrete series representations for the orbit spaces arising from two involutions of real reductive Lie groups, J. Funct. Anal., 152 (1998), 100-135, MR 99c:22012, Zbl 0937.22008.

[18] K. Koufany, Semi-groupe de Lie Associé à une Algèbre de Jordan Euclidienne, Thèse, Université de Nancy, 1993.

[19] K. Koufany and B. Ørsted, Hardy spaces on two-sheeted covering semigroups, J. Lie Theory, 7 (1997), 245-267, MR 98k:22060, Zbl 0884.22006. 
[20] B. Krötz, Formal dimension for semisimple symmetric spaces, Compositio Math., 125 (2001), 155-191, MR 2002b:22024, Zbl 0968.22012.

[21] B.O. Makarevič, Open symmetric orbits of reductive groups in symmetric $R$-spaces, Math. USSR Sbornik, 20 (1973), 406-418, MR 50 \#1170, Zbl 0285.53041.

[22] Yu.A. Neretin, Matrix analogues of the B-function, and Plancherel formula for Berezin kernel representations, Sb. Math., 191 (2000), 683-715, MR 2001k:33030, Zbl 0962.33002.

[23] _ On separation of spectra in anlysis of Berezin kernels, Funct. Anal. Appl., 34 (2000), 197-207, MR 2001m:32012, Zbl 0967.22007.

[24] G. Ólafsson, Fourier and Poisson transformation associated to a semisimple symmetric space, Invent. Math., 90 (1987), 605-629, MR 89d:43011, Zbl 0665.43004.

[25] G.I. Ol'shanski, Invariant cones in Lie algebras, Lie semigroups and the holomorphic discrete series, Funct. Anal. Appl., 15 (1981), 275-285, MR 83e:32032, Zbl 0503.22011.

[26] B. Ørsted, Composition series for analytic continuations of holomorphic discrete series representations of $S U(n, n)$, Trans. Amer. Math. Soc., 260 (1980), 563-573, MR 81g:22020, Zbl 0439.22017.

[27] M. Pevsner, Espace de Bergman d'un semi-groupe complexe, C.R. Acad. Sci. Paris, I (1996), 635-640, MR 97b:22015, Zbl 0843.22002.

[28] W. Schmid, Die randwerte holomorpher funktionen auf hermitischen räumen, Invent. Math., 9 (1969), 61-80, MR 41 \#3806, Zbl 0219.32013.

[29] M. Takeuchi, Polynomial representations associated with symmetric bounded domains, Osaka J. Math., 10 (1973), 441-475, MR 54 \#616, Zbl 0313.32042.

[30] G. Zhang, Berezin transform on line bundles over bounded symmetric domains, J. Lie Theory, 10 (2000), 111-126, MR 2002d:32035, Zbl 0946.43007.

Received December 7, 2000 and revised August 20, 2001.

Department of Mathematics

OKLahoma State University

401 Mathematical SCIEnCES

Stillwater, OK 74078

E-mail address: ssaid@math.okstate.edu 